\title{
Pairwise genetic interactions modulate lipid plasma levels and cellular uptake
}

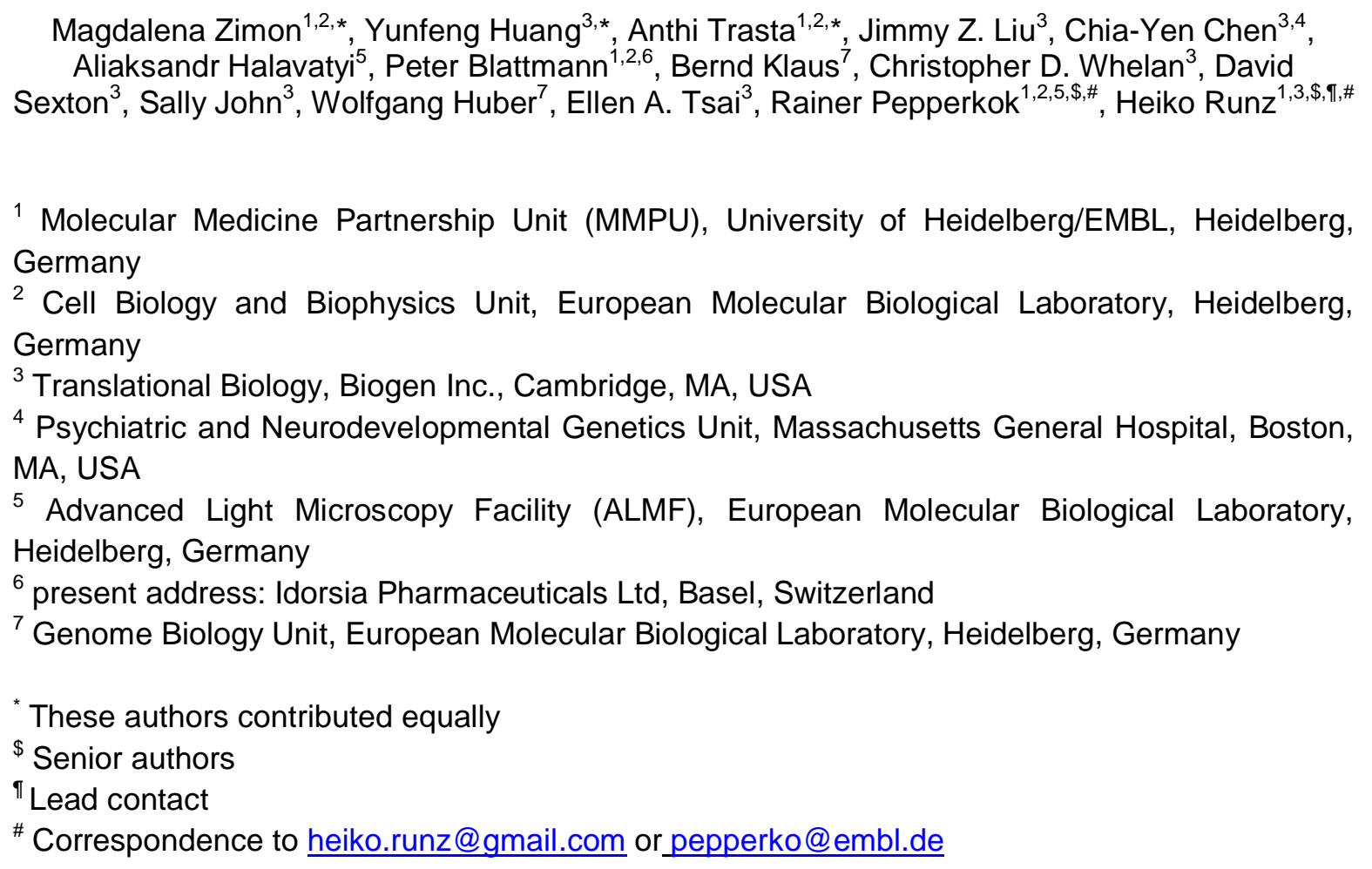

\section{SUMMARY}

Genetic interactions (Gls), the joint impact of different genes or variants on a phenotype, are foundational to the genetic architecture of complex traits. However, identifying Gls through human genetics is challenging since it necessitates very large population sizes, while findings from model systems not always translate to humans. Here, we combined exome-sequencing and genotyping in the UK Biobank with combinatorial RNA-interference (coRNAi) screening to systematically test for pairwise Gls between 30 lipid GWAS genes. Gene-based proteintruncating variant (PTV) burden analyses from 240,970 exomes revealed additive Gls for $A P O B$ with PCSK9 and LPL, respectively. Both, genetics and coRNAi identified additive Gls for 12 additional gene pairs. Overlapping non-additive Gls were detected only for TOMM4O at the APOE locus with SORT1 and NCAN. Our study identifies distinct gene pairs that modulate both, plasma and cellular lipid levels via additive and non-additive effects and nominates drug target pairs for improved lipid-lowering combination therapies. 
bioRxiv preprint doi: https://doi.org/10.1101/2020.10.29.360818; this version posted October 30, 2020. The copyright holder for this preprint (which was not certified by peer review) is the author/funder, who has granted bioRxiv a license to display the preprint in perpetuity. It is made available under aCC-BY-NC-ND 4.0 International license.

40 


\section{INTRODUCTION}

42 Genome-wide association studies (GWAS) have firmly established that changes in blood lipids

43 and the risk of coronary artery disease (CAD) are heritable. Hundreds of genetic loci have been

44 identified that reach genome-wide significant associations with plasma levels of low-density

45 lipoprotein cholesterol (LDL), high-density lipoprotein cholesterol (HDL), triglycerides (TG), total

46 cholesterol (TC) and $\mathrm{CAD}^{1-4}$. In rare instances, susceptibility to altered blood lipids can be

47 attributed to mutations in individual genes such as $L D L R, P C S K 9$ or $A P O B$ that lead to familial

48 forms of disease. For the vast majority of dyslipidemic individuals, however, no single-gene

49 mutation can be identified. Instead, recent evidence suggests that in these cases inherited

50 susceptibility is caused by a cumulative effect of numerous common alleles within and across

51 GWAS loci. Individually, such common alleles have only a minor effect, but when summarized in

52 polygenic scores they can modify a phenotype to a similar extent as single high-impact

53 mutations $^{5}$, or further magnify the penetrance of individual mutations causing Mendelian

54 disease $^{6}$. The biological mechanisms behind the cumulative effect of risk alleles in different

55 genes remain unclear.

56 While the refined understanding of the polygenic nature of complex disease is starting to show

57 promise for improved risk prediction and treatment decisions ${ }^{7,8}$, it has made it increasingly

58 difficult to decide which individual genes could be the most suitable targets for developing new

59 drugs. Drug development is traditionally focused on discrete targets with well-understood

60 biology. For certain diseases, an additive therapeutic benefit has been demonstrated through

61 combination therapies that simultaneously modulate two or more targets at once. For instance,

62 combinations of statins, inhibitors of HMG-CoA-reductase (HMGCR), with distinct other

63 cholesterol-lowering medications including NPC1L1, PCSK9 and APOB inhibitors have been

64 demonstrated to lower LDL levels and CAD-risk further than statin-treatment alone ${ }^{9,10}$. Despite

65 such successes, systematic strategies to predict that joint modulation of drug target pairs in

66 combination therapies will show benefit beyond standard of care have yet to be explored.

67 Genetic support for a drug target increases the probability that a medicine directed against the

68 respective target will succeed by several fold ${ }^{11,12}$. We thus hypothesized that genetics might also 
69 assist in nominating drug target pairs that, when addressed jointly, will have a higher probability

70 to reach a desired therapeutic benefit. A particular attractive approach to prioritize optimal target

71 pairs would be to leverage synergistic gene-gene interactions, where genetic variants in two

72 disease risk genes induce a phenotype that is more pronounced than what would be expected

73 from each of the variants' individual effects. Non-additive genetic interactions (naGls), or

74 epistasis, have been extensively studied in model organisms and cell models with the aim to

75 identify functional relationships among genes and gene products ${ }^{13,14}$. In humans, however, the

76 contribution of naGls to the architecture of complex traits has been controversial. While there is

77 increasing evidence for modifier genes that modulate Mendelian phenotypes in non-additive

78 manners ${ }^{15}$, most of the variance of complex traits appears to be explained by genes acting

79 additively within or between loci (additive Gls, or aGls) ${ }^{16}$.

80 Here we systematically test for pairwise Gls regulating blood lipid levels by studying interactions

81 between 30 genes prioritized based on known lipid-regulatory functions from GWAS loci using

82 three complementary tools: protein-truncating variants (PTVs) identified through exome

83 sequencing in the UK Biobank; reported GWAS lead SNPs genotyped or imputed in the UK

84 Biobank; and combinatorial RNA-interference (coRNAi) screening measuring LDL-uptake into

85 cultured cells. Our combined genetics and functional genomics approach establishes pairwise

86 additive and non-additive Gls as foundational elements in controlling blood lipid levels and

87 highlights distinct gene pairs as promising targets for lipid lowering combination therapies.

88

\section{RESULTS}

\section{Study outline}

91 To explore pairwise interactions between genes in GWAS loci and how these impact plasma lipid

92 levels and LDL-uptake into cultured cells, we followed three parallel approaches: First, we 93 extracted protein-truncating variants (PTVs) from whole exome sequencing data of 200,654

94 participants of the UK Biobank. Second, we utilized GWAS lead SNPs commonly used to

95 construct polygenic risk scores from the full set of 378,033 unrelated participants of European

96 ancestry in the UK Biobank. And third, we conducted systematic RNAi-based combinatorial 
97 knockdown experiments in cells (Figure 1a). We focussed our analyses on 30 high-confidence

98 candidate genes from 18 genomic regions associated with blood lipid levels or the risk for CAD

99 (Table S1). Twenty-eight of these genes had scored as functional regulators of LDL-uptake,

100 cellular levels of free cholesterol, or LDL-receptor (LDLR) mRNA or protein levels in an earlier

101 study where we had functionally analysed 133 genes at 56 lipid and CAD GWAS loci through

102 RNAi-based knockdown experiments ${ }^{17}$. Causality for several of these genes to drive GWAS

103 associations was further supported through systematic colocalization of plasma LDL GWAS lead

104 SNPs with GTEx liver eQTLs ${ }^{1}$ (2 genes), cis-pQTL signals ${ }^{18}$ (3 genes) and independently

105 reported biological evidence for lipid-relevant functions (15 genes) (Table S2). To identify 106 pairwise Gls, we applied four linear regression models (modified from Axelsson et al., $2011^{19}$ ) to

107 model the data. For each gene pair, both the additive genetic interaction effect (aGl) (model 3),

108 which measures the sum of effects from each gene or variant individually, as well as the non-

109 additive genetic interaction effect (naGl) (model 4), which measures the difference between the

110 expected additive and the observed combined effect, were calculated, with a naGl being either

111 synergistic or buffering (Figure 1a and Methods). Pairwise analyses were conducted for four

112 plasma lipid parameters (LDL, HDL, TG, TC) and CAD as available from UK Biobank ${ }^{20}$ (see

113 Methods).

115 PTV burden tests in UK Biobank reveal additive genetic interactions for PCSK9-APOB and

116 LPL-APOB

117 We first studied pairwise modifier effects between the 30 candidate genes using high-impact

118 protein-truncating variants (PTVs). PTVs are expected to cause loss-of-function and compared

119 to other types of mutations are rare at the population level due to purifying selection ${ }^{21,22}$. We

120 sequenced the exomes of 200,654 UK Biobank participants, annotated PTVs using Variant

121 Effect Predictor $v 96^{23}$ and the LOFTEE plugin ${ }^{21}$, and identified 462,762 high-confidence PTVs in

122 the canonical transcripts of 18,869 genes. Within the 30 lipid GWAS genes, we detected a total

123 of 755 unique rare PTVs (Table S3). For instance, we discovered 29 different PTVs in $L D L R, 47$

124 in PCSK9 and 102 in APOB. Most PTVs in these three genes were associated with strongly 
125 abnormal plasma LDL levels in heterozygote carriers consistent with Familial

126 Hypercholesterolemia, although only 32 of the PTVs were annotated as pathogenic or likely

127 pathogenic in $\mathrm{ClinVar}^{24}$.

128 Gene-based PTV-burden association analyses were conducted in a cohort of 161,508 unrelated

129 UK Biobank participants of European ancestry. Single-gene PTV-burden testing identified three

130 genes that were significantly associated (Bonferroni-corrected $p<0.05$ ) with both LDL and TC

131 (APOB, PCSK9, LDLR), two with HDL (LPL, APOB) and two with TG (LPL, APOB), respectively

132 (Table S4). Loss-of-function of these genes had already been identified earlier as associated

133 with the respective lipid traits at the population level ${ }^{2}$. Next, we next expanded from these single

134 gene PTV-burden analyses to study pairwise PTV-based Gls, which could be tested for 42 of the

135435 theoretically possible gene combinations (Table S5 and Methods). For the two gene pairs

136 that met our stringent criteria to be classified as genetic interactions from this analysis, PCSK9-

$137 A P O B$ and $L P L-A P O B$, we conducted replication analyses in an additional 79,462 UK Biobank

138 exomes, bringing the total sample size available for PTV-based GI testing to 240,970 individuals

139 (Table S6). PCSK9-APOB showed an aGI for both, LDL and TC, reflecting that joint loss-of-

140 function of both genes reduces these two lipid measures more than if only one of the two genes

141 is truncated. For instance, PTVs in PCSK9 and APOB individually reduced mean plasma LDL by

$14234.21 \mathrm{mg} / \mathrm{dl}$ and $69.42 \mathrm{mg} / \mathrm{dl}$ relative to individuals without PTVs in these genes, consistent with

143 previous reports ${ }^{25-27}$. However, the three UK Biobank participants who carried both, PCSK9 and

$144 A P O B$ PTVs, showed on average a further reduction in plasma LDL by $40.01 \mathrm{mg} / \mathrm{dl}$ compared to

145 individuals with PTVs in only one of the two genes, and by $90.45 \mathrm{mg} / \mathrm{dl}$ compared to individuals

146 with no PTV in either of the two genes (Figure 1b), suggesting considerable additional protection

147 from CAD. Additive Gls were further identified between $L P L$ and $A P O B$ for $H D L$ and TG.

148 Individuals who carried PTVs in both, $L P L$ and $A P O B$, showed consistently higher HDL and TG

149 levels than individuals with no PTVs, or PTVs in only one gene (Figure 1c). No naGls were

150 identified through PTV-based burden tests in up to 240,970 exomes. Our results are consistent

151 with the prediction that for rare variant-based burden analyses very large sample sizes are

152 necessary to robustly detect Gls in the human population ${ }^{16,25}$. 


\section{Pairwise genetic interactions between GWAS loci modulate plasma lipid levels}

155 We next tested for Gls using 28 lipid/CAD GWAS lead SNPs representing the 30 loci in 378,033 156 unrelated individuals of European ancestry in the UK Biobank ${ }^{20}$. Of a total of 1,890 pairwise 157 SNP-SNP interactions tested, 195, 98, 124, 238 and 10 aGls were identified for LDL, HDL, TG,

158 TC and CAD, respectively (Figure 2a-e; Table S7). Interestingly, SNP-based analyses also 159 suggested pairwise effects between GWAS loci that deviated from an additive model and were 160 classified as naGls. Specifically, we detected ten naGls for LDL, one for HDL, six for TG, and 161 nine for TC (Table 1). No naGl was detected for CAD. The strongest driver of interactions came 162 from the 19q13.32 locus encompassing the CBLC/BCAM/PVRL2/TOMM4O/APOE gene cluster 163 that was contributing to 19 of the 26 naGls identified across all traits. Fourteen naGls were 164 between lead SNPs from within the same GWAS region ("cis-naGl", e.g., NCAN-TM6SF2, 165 BCAM-APOE, ZNF259-SIK3) with nine of them being suggestive cis-effects of rs4420638 near 166 APOE. However, naGls were also identified between loci on different chromosomes ("trans167 naGIs"), such as between ZNF259 and APOE, or SORT1/CELSR2 and TOMM40 for LDL and 168 TC, or between $L P L$ and ZNF259, or LPL and SIK3 for TG. Overall, our data support the 169 hypothesis that aGls between GWAS loci are pervasive and individually small, yet if summed up 170 across many loci in polygenic scores modulate complex traits ${ }^{5}$. Conversely, naGls are 171 considerably less prevalent, with the APOE locus being a potential contributor to naGls for lipid 172 traits.

174 Genetic interactions between gene-based PTV-burden and GWAS loci or polygenic scores

175 Next, we queried for Gls between different types of genetic variation. Pairwise interaction testing 176 between gene-based PTV-burden and GWAS lead SNPs identified one naGl for LDL (LDLR $R_{\mathrm{PTV}^{-}}$ $\left.177 P V R L 2_{\mathrm{SNP}}\right)$, one for HDL (APOB $\left.{ }_{\mathrm{PTV}}-L P L_{\mathrm{SNP}}\right)$, three for TC (LDLR $R_{\mathrm{PTV}}-P V R L 2_{\mathrm{SNP}}, L D L R_{\mathrm{PTV}}-S I K 3_{\mathrm{SNP}}$,

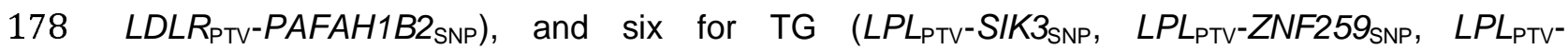
179 PAFAH1B2 $\left.2_{\mathrm{SNP}}, B A Z 1 B_{\mathrm{PTV}}-N C A N_{\mathrm{SNP}}, B A Z 1 B_{\mathrm{PTV}}-T M 6 S F 2_{\mathrm{SNP}}, B A Z 1 B_{\mathrm{PTV}}-P A F A H 1 B 2_{\mathrm{SNP}}\right)$ (Table 180 S8). Moreover, 56, 26, 54 and 31 aGls were identified for LDL, HDL, TC and TG, respectively. 
181 These results are consistent with the genetic architecture regulating plasma lipids being

182 continuous between high-impact rare and low-impact common alleles ${ }^{4}$.

183 A recent study ${ }^{6}$ proposed that the penetrance of Mendelian disease, including $\mathrm{FH}$, can be

184 substantially modulated by interactions between the respective mutant gene with common

185 variants (minor allele frequency $>0.01$ ) of individually small effect size subsumed in polygenic

186 risk scores (PRS). We created PRS for the four lipid species using PRS-CS ${ }^{26}$ (and Methods) and

187 tested for Gls between PRS and PTV-burden for each of the 30 genes. Of all combinations

188 tested, only PTV-burden in LPL, mostly driven by the frequent p.S447Ter variant, showed

189 evidence for a naGl with the PRS for TG $\left(p<1.13 \times 10^{-15}\right.$; beta=-0.04) (Figure 2f; Table S9). This

190 supports the hypothesis that a high polygenic risk for elevated TG can be mitigated by a

191 concomitant stop-gain mutation in LPL. Additionally, 10 aGls were identified between $A P O B_{\mathrm{PTV}}$

192 with PRS for all four lipid species, $L D L R_{\mathrm{PTV}}$ and $P C S K 9_{\mathrm{PTV}}$ with PRS for LDL and TC, and $L P L_{\mathrm{PTV}}$

193 with PRS for LDL and HDL.

195 RNAi identifies pairwise functional gene interactions modulating cellular LDL-uptake

196 To gain insights into the functional consequences of Gls, we complemented our genetic 197 analyses with systematic experiments in cells using combinatorial RNAi (coRNAi) (Figure 3a and

198 Methods). We applied solid-phase reverse transfection to simultaneously knock down candidate

199 gene pairs in cultured HeLa cells, which we have previously shown to reliably reflect various aspects of LDL biology and lipid homeostasis ${ }^{17,27,28}$. Each of the 30 lipid genes was profiled with

201 a single siRNA that had previously been validated to significantly enhance or reduce cellular 202 uptake of fluorescent-labelled LDL (Dil-LDL) or free cellular cholesterol levels, and/or to 203 efficiently downregulate mRNA or protein levels of its respective target gene (Table S2) ${ }^{17}$. The 204 impact of both, single and combinatorial gene knockdown on LDL-uptake per cell was measured 205 and quantified from high-content microscopy images using automated image analysis routines 206 as described (Figure S1) ${ }^{27,28}$. All pairwise knockdown combinations between the 30 lipid genes 207 (435 gene pairs) were assayed in a total of 16,128 experiments (Figure $3 \mathbf{b}$ ). Each combination 208 was tested in at least seven biological replicates. Using BIC-model based robust linear 
209 regression fitting analogous to the genetic interaction analyses, we identified 18 aGls and 33

210 naGls to differentially impact cellular LDL-uptake (Table S10). A similar proportion of Gls was

211 identified using robust linear model fitting and deriving p-values from the linear regression model

212 term describing non-additive effects as an alternative statistical approach (see Methods). This

213 identified 35 naGls, with 31 naGls overlapping between both analytical approaches (Table S11).

214 The corresponding gene pairs were brought forward to independent liquid-phase based coRNAi

215 replication experiments that validated 20 of these naGls (Table 2, Table S12, Figure S2). Of the

21620 validated naGls identified through coRNAi, seven were classified (according to Horlbeck et

217 al., $2018^{14}$ ) as synergistic, i.e., simultaneous knockdown of both genes magnified the effect size

218 beyond expectations for an aGl; and thirteen naGls were categorized as buffering, i.e., relative to

219 an aGl the joint knockdown mitigated LDL-uptake into cells (Figure 3c). For instance,

220 simultaneous knockdown of HMGCR and $A P O B$ enhanced cellular LDL-uptake beyond a mere

221 additive effect expected from knockdown of either of the two genes, proposing a synergistic naGl

222 (Figure 3d), that is most likely explained by a higher capacity of cells to bind and internalize LDL

223 via increased availability of LDL-receptor at the cell surface (Figure S3). Conversely, knockdown

224 of LDLR strongly inhibited, whereas partial knockdown of LDLRAP1 increased cellular LDL-

225 uptake under our experimental conditions. When silencing $L D L R$ and LDLRAP1 jointly, the

226 reduction of LDL-uptake was less attenuated than expected under an additive model, suggesting

227 a buffering naGI (Figure 3e). Interestingly, reduction of LDL-uptake upon knockdown of LDLR

228 was magnified when LDLR was jointly silenced with HAVCR1, a suggested LDL scavenger

229 receptor that might contribute to maintain the potential of LDLR-depleted cells to internalize

$230 \mathrm{LDL}^{29}$ (Figure 3f). Noteworthy, among the remaining validated coRNAi naGls, simultaneous

231 silencing of PCSK9 and TMEM57, as well as of SIK3 and PAFAH1B1 increased cellular LDL-

232 uptake to a similar extent as the simultaneous knockdown of HMGCR and APOB, although

233 silencing of these genes individually had a significant, yet only modest impact on cellular LDL-

234 uptake. In summary, coRNAi identified aGls and naGls between established lipid-regulatory

235 genes, but also proposed combinations of less well characterized genes as potentially important

236 factors in maintaining cellular lipid levels. 
238 Integrated analysis highlights Gls supported by both human genetics and cellular

\section{9 function}

240 In order to assess whether Gls identified through either PTV-based gene-burden tests, GWAS

241 lead SNPs, or cell-based coRNAi overlapped, we integrated results from the three approaches

242 (Figure 4; Table S13). LDLR-SIK3 showed an aGI both in coRNAi and PTV-SNP analyses for

243 LDL (Figure 4a). Both, coRNAi screening $(\triangle \mathrm{BIC} 16.87, \mathrm{pVal}(\mathrm{FDR})=1.18 \mathrm{E}-07)$ and PTV-SNP

244 analyses for LDL and TC proposed a naGl between LDLR and PVLR2 (Figure 4b), although this

245 gene pair failed to score as naGl in the independent coRNAi validation experiments. Twelve of

246 the 18 gene pairs nominated by coRNAi as aGls also scored as aGls in SNP-based interaction

247 testing for LDL and TC, including LDLR-SIK3. Five aGls involved HMGCR and four LDLRAP1

248 (Figure 4c). Two gene pairs, SORT1-TOMM40 and NCAN-TOMM40, scored as naGls both in

249 the SNP-based as well as the coRNAi-based interaction testing (Figure 4d), with TOMM40

250 exerting a buffering naGl in either gene pair (Figure 4e) that could not be explained by an off-

251 target effect of TOMM40 siRNAs on APOE as an adjacent gene in the 19q13.32 GWAS locus

252 (Figure S4). In conclusion, integrating genetic with functional data validated 12 proposed aGls

253 and further substantiates a role of the APOE locus, and possibly TOMM40, as contributing to

254 non-additive genetic interactions.

255

256

\section{DISCUSSION}

257 Here, we apply whole-exome sequencing, genotyping and coRNAi to systematically test for

258 pairwise Gls between 30 lipid-regulatory genes at lipid and CAD GWAS loci. Gls are considered

259 to be central constituents of biological pathways and complex traits, contributors to human

260 disease, and promising starting points for therapy development ${ }^{13,15}$. Mapping Gls, and

261 particularly non-additive epistasis, however, has been challenging. Gl studies require very large

262 population sizes in order to obtain sufficient statistical power, so that the large number of

263 potential interactions to be evaluated quickly leads to a prohibitive number of statistical tests ${ }^{30}$.

264 Together with most Gl studies to date being limited to just a single datatype, the relative 
265 contribution of Gls to variation in human complex traits has been controversial, and the 266 relevance of epistasis potentially overestimated ${ }^{16}$.

267 In our study, we have tried to overcome several of these challenges through a systematic 268 approach to GI testing that integrates genetic with functional data and relies on the UK Biobank, 269 a population cohort linking genetic with phenotype data at an unprecedented scale ${ }^{20}$. To protect 270 against statistical penalties from multiple hypothesis testing we focused on pairwise interaction 271 analyses between 30 candidate genes nominated through GWAS that functional or genetic 272 follow-up studies have proposed as likely causal to confer associations with lipid traits or $C A D^{17}$. 273 We assessed these genes for Gls across the allelic spectrum, from rare PTVs ascertained from 274 the exomes of more than 240,000 individuals, to common GWAS lead SNPs. Genetic Gl-testing 275 was complemented by functionally knocking down gene pairs with siRNAs and determining the 276 consequence on LDL internalization into cells.

277 Several of the Gls identified in our study can be expected to be high potential starting points for 278 the development of advanced lipid-lowering combination therapies. Lowering LDL with statins is 279 the first-line pharmacological strategy to treat or prevent CAD and ischaemic heart disease as its 280 clinical manifestation. However, many patients do not reach their recommended goals of LDL281 lowering through statins alone, or they are intolerant against statins. For these, combination 282 therapies have become available that aim to lower atherogenic lipid levels further. A motivation 283 for this is that every $1 \mathrm{mmol} / \mathrm{l}(39 \mathrm{mg} / \mathrm{dl})$ reduction in blood LDL is associated with a $19 \%$ 284 reduction in coronary mortality and a $21 \%$ reduction in major vascular events, supporting that, at 285 least for secondary prevention, the lower blood LDL levels, the better ${ }^{31}$. Among the options that 286 lower atherogenic blood lipids the most successfully are therapeutics against drug targets that 287 when mutated cause familial hypercholesterolemia $(\mathrm{FH})$, such as NPC1L1, the target of 288 ezetimibe, or $P C S K 9^{9}$. Genetic analyses in extreme phenotypes have identified a small number of individuals with concomitant mutations in two distinct $\mathrm{FH}$ genes, such as $L D L R$ and $A P O B^{32,33}$, $290 L D L R$ and $L D L R A P 1^{34,35}$ or $A P O B$ and $P C S K 9^{36}$. However, due to the rarity of highly penetrant 291 FH mutations such findings have thus far remained limited to individual families. Conversely, on 292 a population level, a previous Gl analysis based on common alleles from $\sim 24,000$ individuals 
293 ascertained for lipid traits reported 14 replicated Gls between lipid GWAS loci, most notably, like 294 in our study, with SNPs at the APOE locus being a key contributor ${ }^{37}$. Additional support for the 295 relevance of Gls for modulating lipid traits comes from a recent study that includes a subset of 296 the UK Biobank exomes analysed here and proposes an interplay of genetic variation across the 297 allelic spectrum ${ }^{6}$. Notably, that study reports that carriers of monogenic CAD risk variants show 298 an up to 12.6-fold higher risk to manifest disease if they are in the highest quintile of the 299 polygenic risk distribution.

300 Our analyses here propose distinct gene pairs that modulate plasma and cellular lipid levels via 301 additive and non-additive Gl effects. Among others, we identify Gls for several prominent 302 cardiovascular risk genes that individually are established targets for lipid-lowering drugs. For 303 instance, coRNAi proposed a synergistic, non-additive Gl between HMGCR, the rate-limiting 304 enzyme during cholesterol biosynthesis and target of statins, and $A P O B$ encoding apolipoprotein $305 \mathrm{~B}$, a critical constituent of LDL particles. Consistent with the known biological functions of these 306 genes, joint knockdown increased levels of functional LDL-receptor on the cell surface and 307 stimulated internalization of exogenous, fluorescent-labelled LDL. This observation is well in line 308 with results from clinical trials showing that in patients with Familial Hypercholesterolemia and 309 other hyperlipidemias a combination of statins with an antisense inhibitor of apolipoprotein B 310 (mipomersen) efficiently reduces plasma LDL levels more strongly than high-intensity statin 311 treatment alone ${ }^{38-41}$. Importantly, the additive GI identified from UK Biobank participants carrying 312 PTVs in both, APOB and PCSK9 suggests that similarly beneficial effects can be expected when 313 APOB antisense therapies are applied in combination with PSCK9 inhibitors. Recently, inclisiran, 314 an siRNA targeting PCSK9 in individuals on maximally tolerated statin doses $^{42}$ led to a 315 persistent, highly significant lowering of LDL in treated individuals relative to placebo in a phase 3163 study $^{43}$, introducing siRNAs as an attractive therapeutic modality for lipid-lowering therapies. 317 Our results strongly propose that, on a population level, combination therapies inhibiting both 318 PCSK9 and $A P O B$ may lower LDL-C levels and CAD-risk even more substantially than drugs 319 targeting only one of the two genes. 
320 APOB PTV-burden was associated not only with LDL and TC, but also HDL and TG, and our

321 PTV-based Gl tests propose that joint disruption of $A P O B$ together with $L P L$ reduces TG and 322 increases HDL, most likely in an additive manner. LPL encodes for lipoprotein lipase which 323 hydrolyzes TG from apolipoprotein B containing lipoproteins, releasing fatty acids ${ }^{44}$. PTV-burden 324 in $L P L$ is dominated by the stop-gain variant p.Ser447Ter (c.1421G>C; rs328) which in our 325 exome-sequenced UK Biobank sub-cohort showed an allele frequency of $9.95 \%$. This variant is 326 known to cause gain-of LPL activity leading to a 0.8 -fold reduced risk for ischaemic heart 327 disease ${ }^{45}$, an effect that is likely to be further enhanced by concomitant reduction of 328 apolipoprotein B. The p.Ser447Ter allele was also the main driver behind the only naGI detected between PTV-burden and polygenic risk for plasma lipids and conferred that in LPL PTV-carriers 330 polygenic risk for TG is reduced, with presumably non-additive effects being the most 331 pronounced in the upper percentile range of the PRS distribution.

332 A prominent driver of Gls in both our SNP- and coRNAi-based analyses was the 19q13.32 locus 333 which includes APOE and apart from plasma lipids and CAD is associated with Alzheimer's 334 disease, longevity and macular degeneration among others ${ }^{18}$. Interestingly, our findings indicate 335 that genes other than APOE at this locus might contribute to lipid Gls, which is consistent with 336 our earlier findings that knock down of several genes at this locus independently modulate 337 cellular LDL-uptake ${ }^{17}$. For instance, both SNP-based GI testing and coRNAi suggested buffering 338 naGls for TOMM40 with SORT1 and NCAN, respectively. Variants in TOMM40 have been 339 hypothesized to modify onset of Alzheimer's disease independently of and in conjunction with $340 A P O E^{45}$. Our analyses suggest TOMM40 might exert similar modifying effects on lipid 341 phenotypes and CAD risk, which will need to be clarified in future studies. Another gene at the $34219 q 13.32$ locus is PVRL2, for which both coRNAi and SNP-PTV analyses proposed Gls with $343 L D L R$. As a vascular cell adhesion molecule, PVRL2 protein regulates transendothelial migration 344 of leukocytes. PVRL2 levels in the atherosclerotic arterial wall correlate with plasma cholesterol 345 in CAD patients and Ldlr-deficient mice and have been linked to the progression of 346 atherosclerosis ${ }^{46,47}$. It is thus tempting to speculate that the extensive pleiotropy of the $19 q 13.32$ 347 locus can at least in part be explained through non-APOE related mechanisms ${ }^{45}$. 
348 Both, genetic and functional analyses further revealed Gls between HAVCR1, NCAN and SIK3

349 with HMGCR, nominating these poorly characterized genes to be explored as potentially

350 attractive new targets for lipid-lowering therapies on top of statins.

351 Consistent with previous assumptions ${ }^{16}$, our results show that for regulating plasma lipid levels,

352 additive Gls between gene or variant pairs are common, while non-additive epistasis is rare.

353 Indeed, despite a sample size of over 240,000 exomes, our gene-based PTV-burden GI

354 analyses did not find evidence for pairwise naGls between lipid genes disrupted by PTVs.

355 Further increasing sample sizes might help uncover non-additive effects, however, at least for

356 lipid traits, their contribution to the overall variance appears to be small. This is consistent with

357 the existence of evolutionary mechanisms that suppress epistatic interactions ${ }^{13}$. Since pairwise

358 naGls can be expected to be identified the most easily for genes that are disrupted sufficiently

359 frequently in a population by PTVs of large-enough effect size, sequencing of consanguineous or

360 bottlenecked populations might improve the detection rate of naGls ${ }^{22,48}$. Interestingly, as

361 observed also here, naGls seem to be more easily detectable in cell and animal models, for

362 instance through synthetic lethality mapping ${ }^{14}$.

363 Integration of population-scale genetics and functional coRNAi screening results yielded a total

364 of twelve aGls and three naGls (one of them suggestive) that influence plasma and cellular lipid

365 levels. Such validation via two systematic approaches substantially increases the confidence for

366 committing to time and resource-intense follow-up analyses of such findings, e.g., when

367 exploring the suitability of a gene pair to be jointly targeted in combination therapies.

368 Interestingly, a significant number of Gls identified through genetics and coRNAi in our study do

369 not yet overlap. This may be explained by several reasons: First, our functional analyses were

370 limited to measuring LDL-uptake into cells, which reflects a relevant, yet only a partial aspect of

371 the many possible mechanisms by which a gene can modulate plasma lipid levels. Second,

372 siRNA-based gene knock down captures acute and rather severe functional effects, which may

373 differ from the chronic and often compensated consequences upon lifelong modulation of a

374 gene's function through genetic variation. Third, despite the large number of samples used for

375 genetics-based Gl testing, the number of informative high-impact variants in the human germline 
376 may still be too discrete to comprehensively identify Gls. Regardless, the availability and rapid

377 development of advanced high-throughput microscopy technology joint with the constantly

378 increasing cohort sizes for genetic analyses will allow up-scaling of the approach taken here in

379 future studies and with a high probability validate further Gls.

380 In conclusion, our study introduces and confirms a strategy to link large-scale genetic data from

381 a population biobank with quantitative, cell-based coRNAi to map Gls that affect blood lipid

382 levels and CAD, an approach that can be applied to other diseases and complex traits. Our

383 unbiased analyses support that mechanisms exist through which multiple genes jointly help

384 maintain blood lipid homeostasis. CAD and ischaemic heart disease remain a substantial global

385 health burden, and doubling-down on lowering atherogenic plasma lipids remains one of the

386 most promising therapeutic approaches. With the encouraging results from recent gene- and

387 antisense-based clinical trials for CAD, our results help prioritize drug target pairs for the 388 development of lipid-lowering combination therapies rooted in human genetics. 


\section{REFERENCES}

391 1. Willer, C., Arbor, A. \& Mohlke, K. Discovery and Refinement of Loci Associated with Lipid $392 \quad$ Levels Supplementary Information. 1-104 (2013) doi:10.1038/ng.2797.

393 2. Liu, D. J. et al. Exome-wide association study of plasma lipids in $>300,000$ individuals. $394 \quad$ Nature Genetics 49, 1758-1766 (2017).

3953 3. Klarin, D. et al. Genetics of blood lipids among $\sim 300,000$ multi-ethnic participants of the Million Veteran Program. Nature Genetics 50, 1514-1523 (2018).

4. Musunuru, K. \& Kathiresan, S. Genetics of Common, Complex Coronary Artery Disease. Cell 177, 132-145 (2019).

5. Khera, A. v. et al. Genome-wide polygenic scores for common diseases identify individuals with risk equivalent to monogenic mutations. Nature Genetics 1 (2018) doi:10.1038/s41588-018-0183-z.

6. Fahed, A. C. et al. Polygenic background modifies penetrance of monogenic variants for tier 1 genomic conditions. Nature Communications 11, 1-9 (2020).

7. Torkamani, A., Wineinger, N. E. \& Topol, E. J. The personal and clinical utility of polygenic risk scores. Nature Reviews Genetics 19, 581-590 (2018).

8. Mars, N. et al. Polygenic and clinical risk scores and their impact on age at onset and prediction of cardiometabolic diseases and common cancers. Nature Medicine 26, 549557 (2020).

9. Ray, K. K. et al. Effect of 1 or 2 Doses of Inclisiran on Low-Density Lipoprotein Cholesterol Levels: One-Year Follow-up of the ORION-1 Randomized Clinical Trial. JAMA Cardiology 4, 1067-1075 (2019).

10. Michos, E. D., McEvoy, J. W. \& Blumenthal, R. S. Lipid management for the prevention of atherosclerotic cardiovascular disease. New England Journal of Medicine 381, 15571567 (2019).

11. Nelson, M. R. et al. The support of human genetic evidence for approved drug indications. Nature Genetics 47, 856-860 (2015).

12. Zheng, J. et al. Phenome-wide Mendelian randomization mapping the influence of the plasma proteome on complex diseases. Nature Genetics (2020) doi:10.1038/s41588-0200682-6.

13. Mackay, T. F. C. Epistasis and quantitative traits: Using model organisms to study genegene interactions. Nature Reviews Genetics 15, 22-33 (2014).

14. Horlbeck, M. A. et al. Mapping the Genetic Landscape of Human Cells. Cell 174, 953967.e22 (2018). 
15. Riordan, J. D. \& Nadeau, J. H. From Peas to Disease: Modifier Genes, Network Resilience, and the Genetics of Health. American Journal of Human Genetics 101, 177191 (2017).

16. Hill, W. G., Goddard, M. E. \& Visscher, P. M. Data and theory point to mainly additive genetic variance for complex traits. PLoS Genetics 4, (2008).

17. Blattmann, P., Schuberth, C., Pepperkok, R. \& Runz, H. RNAi-Based Functional Profiling of Loci from Blood Lipid Genome-Wide Association Studies Identifies Genes with Cholesterol-Regulatory Function. PLoS Genetics 9, (2013).

18. Sun, B. B. et al. Genomic atlas of the human plasma proteome. Nature 558, 73-79 (2018).

19. Axelsson, E. et al. Extracting quantitative genetic interaction phenotypes from matrix combinatorial RNAi. BMC bioinformatics 12, 342 (2011).

20. Bycroft, C. et al. The UK Biobank resource with deep phenotyping and genomic data. Nature 562, 203-209 (2018).

21. Karczewski, K. J. et al. The mutational constraint spectrum quantified from variation in 141,456 humans. Nature 581, 434-443 (2020).

22. Narasimhan, V. M. et al. Humans With Related Parents. Science 352, 474-477 (2016).

23. McLaren, W. et al. The Ensembl Variant Effect Predictor. Genome Biology 17, 1-14 (2016).

24. Landrum, M. J. et al. ClinVar: Improving access to variant interpretations and supporting evidence. Nucleic Acids Research 46, D1062-D1067 (2018).

25. Zuk, O., Hechter, E., Sunyaev, S. R. \& Lander, E. S. The mystery of missing heritability: Genetic interactions create phantom heritability. Proceedings of the National Academy of Sciences 109, 1193-1198 (2012).

26. Ge, T., Chen, C. Y., Ni, Y., Feng, Y. C. A. \& Smoller, J. W. Polygenic prediction via Bayesian regression and continuous shrinkage priors. Nature Communications 10, 1-10 (2019).

27. Bartz, F. et al. Identification of Cholesterol-Regulating Genes by Targeted RNAi Screening. Cell Metabolism 10, 63-75 (2009).

28. Thormaehlen, A. S. et al. Systematic Cell-Based Phenotyping of Missense Alleles Empowers Rare Variant Association Studies: A Case for LDLR and Myocardial Infarction. PLOS Genetics 11, e1004855 (2015).

29. Ichimura, T. et al. Kidney injury molecule-1 is a phosphatidylserine receptor that confers a phagocytic phenotype on epithelial cells. Journal of Clinical Investigation 118, 16571668 (2008).

30. Phillips, P. C. Epistasis - The essential role of gene interactions in the structure and evolution of genetic systems. Nature Reviews Genetics 9, 855-867 (2008). 
461 31. Cholesterol Treatment Trialists' (CTT) Collaborators. Efficacy and safety of cholesterol-

462

463

464

465

466

467

468

469

470

471

472

473

474

475

476

477

478

479

480

481

482

483

484

485

486

487

488

489

490

491

492

493

494

495

496

497

lowering treatment: prospective meta-analysis of data from 90056 participants in 14 randomised trials of statins. The Lancet 366, 1267-1278 (2005).

32. Rauh, G. et al. Identification of a heterozygous compound individual with familial hypercholesterolemia and familial defective apolipoprotein B-100. Klinische Wochenschrift 69, 320-324 (1991).

33. Benlian, P. et al. Phenotypic Expression in Double Heterozygotes for Familial Hypercholesterolemia and Familial Defective Apolipoprotein B-100. 340345, (1996).

34. Tada, H. et al. A novel type of familial hypercholesterolemia: Double heterozygous mutations in LDL receptor and LDL receptor adaptor protein 1 gene. Atherosclerosis 219, 663-666 (2011).

35. Soufi, M., Rust, S., Walter, M. \& Schaefer, J. R. A combined LDL receptor/LDL receptor adaptor protein 1 mutation as the cause for severe familial hypercholesterolemia. Gene 521, 200-203 (2013).

36. Elbitar, S. et al. New Sequencing technologies help revealing unexpected mutations in Autosomal Dominant Hypercholesterolemia. Scientific Reports 8, 1-10 (2018).

37. De, R. et al. Identifying gene-gene interactions that are highly associated with four quantitative lipid traits across multiple cohorts. Human Genetics 136, 165-178 (2017).

38. Akdim, F. et al. Effect of Mipomersen, an Apolipoprotein B Synthesis Inhibitor, on LowDensity Lipoprotein Cholesterol in Patients With Familial Hypercholesterolemia. American Journal of Cardiology 105, 1413-1419 (2010).

39. Akdim, F. et al. Efficacy of apolipoprotein B synthesis inhibition in subjects with mild-tomoderate hyperlipidaemia. European Heart Journal 32, 2650-2659 (2011).

40. McGowan, M. P. et al. Randomized, Placebo-Controlled Trial of Mipomersen in Patients with Severe Hypercholesterolemia Receiving Maximally Tolerated Lipid-Lowering Therapy. PLoS ONE 7, 1-10 (2012).

41. Fogacci, F. et al. Efficacy and Safety of Mipomersen: A Systematic Review and MetaAnalysis of Randomized Clinical Trials. Drugs vol. 79 751-766 (2019).

42. Fitzgerald, K. et al. A highly durable RNAi therapeutic inhibitor of PCSK9. New England Journal of Medicine 376, 41-51 (2017).

43. Raal, F. J. et al. Inclisiran for the treatment of heterozygous familial hypercholesterolemia. New England Journal of Medicine 382, 1520-1530 (2020).

44. Merkel, M., Eckel, R. H. \& Goldberg, I. J. Lipoprotein lipase: Genetics, lipid uptake, and regulation. Journal of Lipid Research 43, 1997-2006 (2002).

45. Roses, A. et al. Understanding the genetics of APOE and TOMM40 and role of mitochondrial structure and function in clinical pharmacology of Alzheimer's disease. Alzheimer's and Dementia 12, 687-694 (2016). 
498

499

500

501

502

503

504

505

506

507

508

509

510

511

512

513

514

515

516

517

518

519

520

521

522

523

524

525

526

527

528

529

530

531

53261.

53362

534

46. Björkegren, J. L. M. et al. Plasma Cholesterol-Induced Lesion Networks Activated before Regression of Early, Mature, and Advanced Atherosclerosis. PLoS Genetics 10, (2014).

47. Rossignoli, A. et al. Poliovirus Receptor-Related 2: A Cholesterol-Responsive Gene Affecting Atherosclerosis Development by Modulating Leukocyte Migration. Arteriosclerosis, Thrombosis, and Vascular Biology 37, 534-542 (2017).

48. Locke, A. E. et al. Exome sequencing of Finnish isolates enhances rare-variant association power. Nature 572, 323-328 (2019).

49. van der Harst, P. \& Verweij, N. Identification of 64 Novel Genetic Loci Provides an Expanded View on the Genetic Architecture of Coronary Artery Disease. Circulation Research 122, 433-443 (2018).

50. Kamat, M. A. et al. PhenoScanner V2: An expanded tool for searching human genotypephenotype associations. Bioinformatics 35, 4851-4853 (2019).

51. Burton, P. R. et al. The Wellcome Trust Case Control Consortium. Genome-wide association study of 14,000 cases of seven common diseases and 3,000 shared controls. Nature 447, 661-678 (2007).

52. Lonsdale, J. et al. The Genotype-Tissue Expression (GTEx) project. Nature Genetics 45, 580-585 (2013).

53. Giambartolomei, C. et al. Bayesian Test for Colocalisation between Pairs of Genetic Association Studies Using Summary Statistics. PLoS Genetics 10, (2014).

54. Hout, C. V. van et al. Whole exome sequencing and characterization of coding variation in 49,960 individuals in the UK Biobank. bioRxiv 572347 (2019) doi:10.1101/572347.

55. Schwarz, G. Estimating the Dimension of a Model. Ann. Statist. 6, 461-464 (1978).

56. Erfle, H. et al. Reverse transfection on cell arrays for high content screening microscopy. Nature protocols 2, 392-399 (2007).

57. Erfle, H. et al. Work flow for multiplexing siRNA assays by solid-phase reverse transfection in multiwell plates. Journal of biomolecular screening $\square$ : the official journal of the Society for Biomolecular Screening 13, 575-580 (2008).

58. Carpenter, A. E. et al. CellProfiler: Image analysis software for identifying and quantifying cell phenotypes. Genome Biology 7, (2006).

59. Gilbert, D. F., Meinhof, T., Pepperkok, R. \& Runz, H. DetecTiffC: A novel image analysis routine for high-content screening microscopy. Journal of Biomolecular Screening 14, 944-955 (2009).

60. Malo, N., Hanley, J. A., Cerquozzi, S., Pelletier, J. \& Nadon, R. Statistical practice in high-throughput screening data analysis. Nature Biotechnology 24, 167-175 (2006).

1. Birmingham, A. et al. Interference Screens. Nature Methods 6, 569-575 (2010).

2. Raftery, A. E. Bayesian Model Selection in Social Research. Sociological Methodology 25, 111 (1995). 
bioRxiv preprint doi: https://doi.org/10.1101/2020.10.29.360818; this version posted October 30,2020 . The copyright holder for this preprint (which was not certified by peer review) is the author/funder, who has granted bioRxiv a license to display the preprint in perpetuity. It is made available under aCC-BY-NC-ND 4.0 International license.

535 63. Yoav Benjamini \& Yosef Hochberg. Controlling the False Discovery Rate: A Practical and Powerful Approach to Multiple Testing. Journal of the Royal Statistical Society. Series $B$ (Methodological) 57, 289-300 (1995).

538 


\section{ACKNOWLEDGEMENTS}

540 This research has been conducted using the UK Biobank resource under application number

541 26041. We thank all the participants and researchers of UK Biobank for making these data open

542 and accessible to the research community. AbbVie, Anylam Pharmaceuticals, AstraZeneca,

543 Biogen, Bristol-Myers Squibb, Pfizer, Regeneron and Takeda are acknowledged for generation

544 and initial quality control of the whole-exome sequencing data. We thank Eric Marshall,

545 Yongsheng Huang and Frank Nothaft for infrastructure support for genetic data analyses. The

546 EMBL Advanced Light Microscopy Facility is acknowledged for supporting high-content

547 microscopic-based screening analyses. We are grateful to Brigitte Joggerst, Susanne Theiss and

548 Miriam Reiss for excellent technical assistance. Support to the study came in part from the

549 Transatlantic Networks of Excellence Program 10CVD03 from Fondation Leducq to HR and RP.

$550 \mathrm{MZ}$ was supported by the EMBL EIPOD programme, AT and PB by the EMBL PhD programme.

551

552 AUTHOR CONTRIBUTIONS

553 Conceptualization, H.R. and R.P.; Methodology and Investigation, M.Z., Y.H., A.T., C.-YC., R.P.

554 and H.R.; Formal Analysis and Validation, M.Z., Y.H., A.T., C.-Y.C., J.L., A.H., B.K., E.T. and

555 H.R.; Resources and Data Curation, J.L., P.B., C.W., D.S., S.J., E.T., R.P. and H.R.; Writing -

556 Original Draft, M.Z., A.T. and H.R; Writing - Review and Editing, M.Z., Y.H., P.B., E.T., R.P. and

557 H.R.; Supervision, S.J., W.H., E.T., R.P. and H.R.; Project Administration and Funding

558 Acquisition, S.J., R.P. and H.R.

559

560 DECLARATION OF INTERESTS

561 Y.H., C.-Y.C., J.L., C.W., D.S., S.J., and H.R. are full-time employees at Biogen, Inc. The funders

562 had no role in study design, data collection and analysis, decision to publish, or preparation of

563 the manuscript.

564

565 Further information and requests for resources and reagents should be directed to and will be 566 fulfilled by the Lead Contact, Heiko Runz (heiko.runz@gmail.com) 


\section{METHODS}

\section{Gene Selection}

569 We chose to study 30 candidate genes from 18 loci reported as associated through common570 variant genome-wide association studies (GWAS) as associated with plasma lipid levels and the 571 risk for CAD. Twenty-eight of these genes had been identified and validated as functional 572 regulators of LDL-uptake and/or cholesterol levels into cells in a previous RNAi-screen analysing 573 a total of 133 genes in 56 lipid and CAD GWAS loci ${ }^{17}$ (Table S1). Common-variant association 574 signals and published biological evidence for potential roles in lipid regulation were updated for

575 all 30 candidate genes based on the recent literature (e.g., ${ }^{1-3,49}$ ) and queries using the 576 PhenoScanner platform ${ }^{50}$ (http://www.phenoscanner.medschl.cam.ac.uk/). Twenty eight genes 577 were validated to reside within loci that are associated at genome-wide significance $(p<5 e-8)$ 578 with plasma lipid levels or CAD. SNPs near FAM174A (rs383830) and SEZ6L (rs688034) had 579 originally been reported as associated with $C A D^{51}$, but failed to replicate at genome-wide 580 significance in more recent meta-GWAS. However, since knockdown of both genes had scored 581 as significantly impacting lipid parameters in cells ${ }^{17}$ the two genes were maintained for this 582 current study.

583

584 Colocalization Analysis

585 Colocalization analysis was performed between the 28 GWAS lead SNPs using summary 586 statistics from the 2013 Global Lipid Genetics Consortium GWAS 587 (http://csg.sph.umich.edu/willer/public/lipids2013/) and the GTEx liver cis-eQTL dataset $588(\mathrm{~N}=153)^{52}$. When a respective locus was associated with multiple lipid phenotypes, the SNP with 589 the lowest reported p-value association with LDL was chosen to be the lead SNP. There was no 590 GTEx liver expression data for four genes (APOE, MYBPHL, NCAN, SEZ6L), therefore there 591 were no cis-eQTL for these genes to colocalize with. Colocalization analysis was conducted 592 following the methods in Giambartolomei et al., $2014^{53}$ using the R 'coloc' package on a $+/-500 \mathrm{~kb}$ 593 window around each lead SNP against SNP-to-expression data of all neighbouring genes within 594 that locus. Positive colocalization between liver cis-eQTL and GWAS signal was defined as 
595 showing a posterior probability of sharing the same SNP (PP4) if larger than 0.8. A lead SNP at

596 the SORT1/CELSR2 locus (rs629301) showed a positive colocalization signal, but the cis-eQTL

597 co-localized with both genes, so SNP-based Gls for these genes could not be analysed

598 separately.

599

600 UK Biobank lipid and CAD phenotypes

601 The UK Biobank is a prospective study of over 500,000 participants recruited at an age of $40-69$

602 years from 2006-2010 in the United Kingdom. Participant data include health records, medication

603 history and self-reported survey information, together with imputed genome-wide genotypes and

604 biochemical measures ${ }^{20}$. Baseline biochemical measures including LDL cholesterol (LDL), HDL

605 cholesterol (HDL), triglycerides (TG), and serum total cholesterol (TC) had been obtained in UK

606 Biobank's purpose-built facility in Stockport as described in the UK Biobank online data

607 showcase and protocol (www.ukbiobank.ac.uk). Demographic and other relevant phenotypic

608 information was obtained from standard questionnaire data. Individual lipid phenotypes (LDL,

$609 \mathrm{HDL}, \mathrm{TG}$ and TC) were modelled as dependent variables using linear regression models against

610 covariates including age, sex, smoking, alcohol drinking status, and BMI. Lipid medication use

611 was obtained from self-reported questionnaire data (UK Biobank fields 6153 and 6177). CAD

612 cases were recognized based on both self-reported diagnosis and Hospital Episode Statistics

613 data in the UK Biobank with a code-based CAD definition as presented in the most recent CAD

614 GWAS that included UK Biobank ${ }^{49}$. In total, 30,125 CAD cases were identified and the cohort

615 was adjusted for age, sex, smoking status, alcohol drinking status, BMI and lipid medication use.

616 All phenotype data were derived from UK Biobank basket "ukb27390" released on March 11,

6172019.

618

619 Pairwise gene-based PTV-burden interaction testing

620 High-impact protein-truncating variants (PTVs) expected to disrupt protein functions were

621 identified from 200,654 whole-exome sequencing (WES) data of UK Biobank participants to 622 conduct pairwise interaction analyses. WES data was generated and quality controlled (QC-ed) 
623 as described in Van Hout et al. at the Regeneron Genetics Center as part of a collaboration 624 between AbbVie, Alnylam Pharmaceuticals, AstraZeneca, Biogen, Bristol-Myers Squibb, Pfizer, 625 Regeneron and Takeda and the UK Biobank consortium ${ }^{54}$. PTVs were called from a Regeneron 626 QC-passing "Goldilocks" set of genetic variants using Variant Effect Predictor v96 ${ }^{23}$ (McLaren et 627 al., 2016) and the LOFTEE plugin ${ }^{21}$. We identified 462,762 high-confidence PTVs with a minor 628 allele frequency of $<1 \%$ in the canonical transcripts of 18,869 genes. This set included 755 rare 629 PTVs in the 30 lipid genes analysed in this study. PTVs per gene were enumerated, and a PTV630 burden association analysis was conducted in 161,508 unrelated ( $>2^{\text {nd }}$ degree relatedness) UK 631 Biobank participants of European ancestry, as defined by principle components analysis of the 632 genotyping data ${ }^{20}$. Replication analysis was conducted from an additional 101,827 samples, 633 bringing the total sample size used for calling PTVs from UK Biobank exome sequencing data to 634302,634 . Of these 101,827 samples, 79,462 fulfilled the criteria applied to the discovery cohort, 635 so that an overall sample size of 240,970 exomes was available for replicating findings from the 636 initial PTV-based Gl analyses.

637 For pairwise PTV-based interaction testing, QC-ed UK Biobank lipid phenotypes (HDL, LDL, TG 638 and TC) were modelled as dependent variables using the following four linear regression models 639 in R:

640 Model 1 for gene1 PTV-burden only: lipids $\sim$ PTV 1

641 Model 2 for gene2 PTV-burden only: lipids $\sim$ PTV $_{2}$

642 Model 3 for gene1 PTV-burden and gene2 PTV burden (additive GI): lipids PTV $1+$ PTV $_{2}$

643 Model 4 for gene1 PTV-burden and gene2 PTV burden (non-additive G): lipids $\sim$ PTV $_{1}+$ PTV $_{2}+$ $644 \mathrm{PTV}_{1}{ }^{*} \mathrm{PTV}_{2}$

645 Schwarz's Bayesian Information Criterion $(\mathrm{BIC})^{55}$ scoring was used to determine the best model 646 to explain the data and goodness of fit, with the lowest BIC value indicating the best-fitting model 647 describing each gene pair. Model 3 reflected additive genetic interactions (aGls), Model 4 non648 additive gene interactions (naGls). The model with the lowest BIC was chosen as describing 649 most adequately the type of interaction between each corresponding gene pair. 


\section{Pairwise SNP interaction testing}

652 To assess whether GWAS lead SNPs modulate plasma lipid levels through joint effects within

653 and across GWAS loci, we conducted pairwise SNP-SNP interaction analysis using genome-

654 wide genotyping data and biochemical measures of lipid species from the UK Biobank. Twenty-

655 eight lead SNPs mapped to the 30 lipid GWAS genes were extracted from genotyping data of

656378,033 unrelated (removed up to $2^{\text {nd }}$ degree relatedness) participants of European ancestry. A

657 total of 378 pairwise modifier effects were tested by conducting Robust Linear Model Fitting

658 using R, running the following four linear regression models:

659 Model 1 for SNP1 only: lipids $\sim$ SNP $_{1}$

660 Model 2 for SNP2 only: lipids $\sim \mathrm{SNP}_{2}$

661 Model 3 for SNP1 and SNP2 (additive Gl): lipids $\sim \mathrm{SNP}_{1}+\mathrm{SNP}_{2}$

662 Model 4 for SNP1 and SNP2 (non-additive Gl): lipids $\sim \mathrm{SNP}_{1}+\mathrm{SNP}_{2}+\mathrm{SNP}_{1}{ }^{*} \mathrm{SNP}_{2}$

663 Schwarz's Bayesian Information Criterion (BIC) scoring was used to determine the best model to

664 explain the data and goodness of fit, with the lowest BIC value indicating the best-fitting model

665 describing each SNP pair. If Model 3 had the lowest BIC value, it reflected an aGI, and if Model 4

666 had the lowest BIC value, it reflected a naGI.

667 A similar strategy was applied for pair-wise interaction testing to explore potential joint effects

668 between the 30 genes on CAD risk by running the following four logistic regression models

669 adjusted for age, sex, smoking status, alcohol drinking status, BMI and lipid medication use:

670 Model 1 for SNP1 only: CAD SNP 1

671 Model 2 for SNP2 only: CAD SNP 2

672 Model 3 for SNP1 and SNP2 (additive Gl): CAD $\mathrm{SNP}_{1}+\mathrm{SNP}_{2}$

673 Model 4 for SNP1 and SNP2 (non-additive Gl): CAD $\sim \mathrm{SNP}_{1}+\mathrm{SNP}_{2}+\mathrm{SNP}_{1}{ }^{*} \mathrm{SNP}_{2}$

674 As above, the model with the lowest BIC was chosen as describing most adequately the type of

675 interaction between each corresponding SNP pair.

676

677 PTV-SNP interaction testing 
678 In order to conduct pairwise interaction analyses between GWAS lead SNPs and PTVs, we

679 assessed the interaction of the 28 lead SNPs with rare PTV burden for each of the 30 genes. For

680 SNP-PTV interaction testing, UK Biobank lipid phenotypes (HDL, LDL, TG and TC) were

681 modelled as dependent variables using the following four linear regression models:

682 Model 1 for gene1 lead SNP only: lipids $\sim \mathrm{SNP}_{1}$

683 Model 2 for gene2 PTV-burden only: lipids PTV 2

684 Model 3 for gene1 lead SNP and gene2 PTV burden (additive Gl): lipids $\sim \mathrm{SNP}_{1}+\mathrm{PTV}_{2}$

685 Model 4 for gene1 lead SNP and gene2 PTV burden (non-additive Gl): lipids $\sim \mathrm{SNP}_{1}+\mathrm{PTV}_{2}+$ $686 \mathrm{SNP}_{1}{ }^{*} \mathrm{PTV}$

687 As above, the model with the lowest BIC was chosen as describing most adequately the type of 688 interaction between each corresponding SNP-gene pair.

689

\section{PTV-PRS interaction testing}

691 We assessed the interaction effects between polygenic risk score (PRS) and PTVs for each of 692 the four lipid phenotypes. To construct PRS for UK Biobank samples, we first derived the PRS 693 weights for each SNP across the genome using PRS-CS ${ }^{26}$, which is a Bayesian regression694 based algorithm, and publicly available summary statistics from lipid GWAS ${ }^{1}$. We applied derived 695 PRS weights to imputed genotypes (with minor allele frequency $>0.01$ and imputation quality 696 INFO >0.8) of UK Biobank samples and calculated PRS for each lipid, based on the 697 corresponding PRS weights. Note that all SNPs in the gene of interest were excluded from the 698 PRS when testing for PRS-PTV gene interaction. Gls were tested between PRS and PTV699 burden for each of the 30 genes by fitting the four linear regression models:

700 Model 1 for PRS only: lipids PRS

701 Model 2 for gene PTV-burden only: lipids PTV

702 Model 3 for PRS and gene PTV burden (additive Gl): lipids PRS + PTV

703 Model 4 for PRS and gene PTV burden (non-additive Gl): lipids PRS + PTV + PRS * PTV

704 As above, the model with the lowest BIC was chosen as describing most adequately the type of 705 interaction between each corresponding PRS-gene pair. 


\section{RNAi interaction testing}

\section{Cells and reagents}

709 HeLa-Kyoto cells are a strongly adherent Hela isolate (gift from S. Narumiya, Kyoto University

710 Japan) that, as we demonstrated earlier, enable reliable measurements of LDL-cholesterol

711 uptake dynamics and show lipid homeostatic mechanisms similar to those described for liver-

712 derived cell models ${ }^{17,27,28}$. Dil-LDL (Life Technologies), DRAQ5 (Biostatus), Dapi (Molecular

713 Probes), 2-hydroxy-propyl-beta-cyclodextrin (HPCD) (Sigma), Lipofectamine 2000 (Invitrogen)

714 and Benzonase (Novagen) were purchased from the respective suppliers.

715

\section{6 siRNA selection and production of siRNA microarrays}

717 RNA-interference (RNAi) screening was conducted in glass-bottomed single-well chambered cell

718 culture (Lab-Tek) slides with solid-phase reverse siRNA-transfection of cultured cells ("cell

719 microarrays") as described previously ${ }^{27,56}$. Each gene under study was targeted with a single

720 siRNA (Silencer Select, Invitrogen) that had been selected with the EMBL-generated software

721 tool bluegecko (J.K. Hériche, unpublished) based on the alignment to the reference genome, a

722 maximal number of protein-coding transcripts per gene targeted and expected specificity for the

723 target gene. The 28 siRNAs in this study had been validated earlier to significantly enhance or

724 reduce cellular uptake of fluorescent-labelled LDL (Dil-LDL) or free cellular cholesterol levels ${ }^{17}$

725 and were shown to efficiently downregulate mRNA or protein levels of their respective target

726 genes (Table S2). siRNA sequences are provided in Blattmann et al., 2013 Supplementary

727 Table 4. For the two genes not analysed in our earlier study (MYLIP, PAFAH1B2), siRNAs used

728 in the current study were prioritized from 3 and 5 siRNAs per gene based on bluegecko in silico

729 analyses, knockdown efficiency on target mRNA/protein levels (up to less than $10 \%$ residual

730 levels) and/or efficiency to modulate cellular Dil-LDL uptake in preparatory individual single gene

731 knock-down experiments (not shown). The $75 \%(12 / 16)$ of siRNAs that had scored as

732 individually modulating cellular Dil-LDL uptake in our earlier study ${ }^{17}$ also met the more stringent

733 criteria of our current study to score as LDL-uptake modulator when used either alone or 
734 together with non-silencing control siRNA Neg9 (Figure 3b, CTRL column), thereby replicating

735 our earlier results and validating experimental settings for this current study.

736 To cover the total of 435 pairwise siRNA combinations including controls and replicas, five

737 different cell microarrays with 384 spots/array were produced. Per array, the following negative

738 controls were added: eight spots containing INCENP-siRNA (s7424) to control for transfection

739 efficiency ${ }^{17}$; eight spots containing non-silencing control siRNA Neg1 (s229174), and eight spots

740 containing non-silencing control siRNA (denoted as CTRL throughout the text) Neg9 (s444246).

741 Furthermore, eight spots were added with siRNA targeting $L D L R$ (s224006) as a positive control

742 for LDL uptake, as well as eight spots with siRNA targeting NPC1 (s237198) knockdown of

743 which increases free cellular cholesterol signals ${ }^{27}$. For pairwise combinatorial RNAi-screening,

744 siRNAs against two genes were printed simultaneously on a respective siRNA-spot, with equal

745 amounts (15 pmol/siRNA) of siRNA per gene. As positive controls, eight spots containing both,

746 non-silencing control siRNA Neg9 (CTRL) (s444246) and siRNA targeting LDLR (s224006), and

747 eight spots containing both, non-silencing control siRNA (CTRL) Neg9 (s444246) and siRNA

748 targeting NPC1 (s237197) were included per array. For all genes, "single-gene knockdown"

749 scenarios [siRNA geneA $+\mathrm{Neg} 9$ ] were added on two spots per array. Each pairwise "combinatorial

750 knockdown" scenario [siRNA geneA $\left.+\operatorname{siRNA}_{\text {geneB }}\right]$ was analyzed on one spot per array, with a single

751 spot covering 50-100 informative cells ${ }^{28,57}$ (Figure S1).

752 In order to confirm Gls identified with the coRNAi screen, we replicated our analyses with

753 forward transfection in a liquid-phase format with Lipofectamine 2000 reagent in 12-well plates,

754 according to the manufacturer's instructions. Concentrations of the siRNAs were adjusted to

755 mimic the single knockdown phenotypes from the screen (Table S2). $1 \mu$ l of Lipofectamine 2000

756 was used per each transfection. Gls that showed a statistically significant interaction effects

$757\left(p_{\mathrm{fdr}}<10^{-2}\right)$ in replication analyses and acted in the same direction (same directionality of

758 interaction value) as in the coRNAi screen, were considered as validated (Table S12).

759

760 Cell culture, transfection and LDL-uptake assay

761 HeLa Kyoto cells were grown in DMEM medium (Gibco) supplemented with $10 \%$ (w/v) fetal calf 
762 serum (FCS)(PAA) and $2 \mathrm{mM} \mathrm{L-glutamine} \mathrm{(Sigma)} \mathrm{at} 37{ }^{\circ} \mathrm{C}$ with $5 \% \mathrm{CO}_{2}$ and saturated

763 humidity. Cells were plated at a density of $6 \times 10^{4}$ per plate on the cell microarrays for solid-phase

764 siRNA transfection ${ }^{56}$ and cultivated for 48 hours before performing the LDL-uptake assay. For

765 liquid phase transfection-based validation experiments, cells were plated in 12-well plates the

766 day prior to transfection, and siRNA-transfected cells were cultivated for 48 hours. The assays to

767 monitor cellular uptake of fluorescently-labelled LDL (Dil-LDL) were performed as described in

768 more detail in previous publications ${ }^{17,27,28}$. In brief, cells cultured in serum-free medium

769 (DMEM/2mM L-glutamine/0.2 \% (w/v) BSA) and exposed to 1\% 2-hydroxy-propyl-beta-

770 cyclodextrin for $45 \mathrm{~min}$ were labelled with $50 \mu \mathrm{g} / \mathrm{ml}$ Dil-LDL (Invitrogen) for $30 \mathrm{~min}$ at $4{ }^{\circ} \mathrm{C}$. Dil-

771 LDL uptake was stimulated for $20 \mathrm{~min}$ at $37.0^{\circ} \mathrm{C}$ before washing off non-internalized dye for 1

772 min in acidic ( $\mathrm{pH}$ 3.5) medium at $4{ }^{\circ} \mathrm{C}$, fixation, and counterstaining for nuclei (Dapi) and cell

773 outlines (DRAQ5). For RNAi-based gene interaction screening, each of the five cell microarrays

774 was assayed in 7-10 biological replicas.

775

776

Image acquisition and quality control

777 Image acquisition was performed using an Olympus IX81 automated microscope with Scan^R

778 software and an UPlanSApo 20x/NA 0.40 air objective as described ${ }^{17,28}$. Images from a total of

77942 cell microarrays were visually quality controlled. Arrays with insufficient knockdown efficiency

780 where INCENP siRNA treated cells did not show the expected multinucleated phenotype in the

781 DAPI channel were excluded. Also arrays with plate effects as evaluated through diagnostic

782 plots with the splot function in $\mathrm{R}$, and arrays where knockdown of $L D L R$, or $L D L R$ together with

783 negative control siRNA Neg9, did not show a significant difference from controls, were discarded

784 as well. Following these QC criteria, 29 cell microarrays with a total of 11,047 image frames per

785 channel were further analysed. The in-house developed tool HTM Explorer (Ch. Tischer;

786 https://github.com/embl-cba/shinyHTM) was then used to select images fulfilling pre-defined

787 criteria for cell number, image sharpness quality, and image background intensity, resulting in a

788 total number of $9,539(86.35 \%)$ QC-ed image frames that were used for subsequent analyses. 


\section{$790 \quad$ Image analysis}

791 Automated image analysis was performed using a specifically developed pipeline (available

792 upon request) in the open source software CellProfiler ${ }^{58}$ http://www.cellprofiler.org as 793 described ${ }^{17,28}$. In brief, areas of individual cells were approximated by stepwise dilation of masks 794 on the DAPI (nuclei) and DRAQ5 (cell outlines) channels ${ }^{59}$. For each individual cell, Dil-LDL

795 signal was determined from masks representing intracellular endosome-like vesicular areas that 796 were determined by local adaptive thresholding according to predefined criteria for size and 797 shape (Figure S1). Total fluorescence intensity of Dil-signal above local background per cell 798 mask was quantified, and means were calculated from all cells per image. Then, for each siRNA, 799 or siRNA combination ("treated"), signals from different images from the same biological replicate

800 were averaged and a robust Z-score was calculated using the median fluorescence signal of all 801 the negative control siRNAs per array ("median(controls)") and by the median absolute deviation 802 of these controls ("mad(controls)") as follows:robust $Z-$ score $=\frac{I_{F} \text { treated-medianI } I_{F}(\text { controls })}{\operatorname{mad}_{F}(\text { controls })}{ }^{60,61}$. A 803 median robust Z-score was calculated per treatment across all biological replicates and is 804 represented on the plots.

805

806 RNAi gene interaction testing

807 To identify pairs of genes for which simultaneous knock-down results in an additive or non808 additive gene interaction effects on LDL uptake we conducted a Robust Linear Model fitting in R.

809 RobustZScore values calculated from different biological replicates in the presence of single 810 ([siRNA $\left.A_{\text {geneA }}+\mathrm{Neg} 9\right]$ and $\left.\left[\mathrm{siRNA}_{\text {geneB }}+\mathrm{Neg} 9\right]\right)$ or double knock-downs ([siRNA $\left.\left.\mathrm{geneA}+\operatorname{siRNA}_{\text {geneB }}\right]\right)$

811 were considered to be response variable value. Negative control values [Neg9] were included in 812 each fitted dataset to correctly account for baseline LDL uptake. The full regression model 813 considered in the study was

$814 y=\beta_{0}+\beta_{A}{ }^{*} X_{A}+\beta_{B}{ }^{*} X_{B}+\beta_{A B}{ }^{*} X_{A}{ }^{*} X_{B}+\square$

815 which is equivalent to the short form of the statistical formula:

816 $y \sim x_{A}+x_{B}+x_{A}{ }^{*} x_{B}$ 
817 In both formulas $y$ corresponds to the robustZscore values of measured LDL uptake; $x_{A}, x_{B}$ are

818 encoded predictor variables, which are equal to 1 in case of presence of $\operatorname{siRNA} A_{\text {geneA, }}$, siRNA $A_{\text {geneB }}$,

819 or both siRNAs accordingly and equal 0 otherwise. The $\square$ is a noise term, which is minimised

820 during the fitting process. Model fitting provides estimates of $\beta_{0}, \beta_{A}, \beta_{B}$ and $\beta_{A B}$ values. $\beta_{0}$ defines

821 the effect of the negative control on robustZscore values and can be also denoted as an

822 intercept of the linear fit. For our data $\beta_{0}$ is always close to 0 because of the robustZscore

823 definition. The $\beta_{A}$ and $\beta_{B}$ define individual effects of siRNA $A_{\text {geneA }}$ and siRNA $A_{\text {geneB }}$ accordingly. The

$824 \beta_{A B}$ defines the interaction effect between genes $A$ and $B$ and represents the difference between

825 the observed robustZscore values in case of double knockdown $\mathrm{y}_{\mathrm{AB}}$ and the expected additive

826 effect of gene $A$ and geneB knockdown $\left(\beta_{A B}=y_{A B}-\beta_{0}-\beta_{A}-\beta_{B}\right)$.

827 Subsequently, two strategies were used to evaluate functional interactions for each gene pair

828 using defined statistical model:

829 First, to determine gene pairs for which genetic interactions and additive effects observed upon 830 combinatorial knockdowns, we compared fitting of the whole model to the fitting of reduced

831 model versions. Following models were compared:

832 Model 0 - (only baseline effect $\beta_{0}$ in case of either single or double knockdown): $y \sim 1$

833 Model 1 - effect of siRNA geneA $_{\text {only: }} \mathrm{y} \sim \mathrm{x}_{\mathrm{A}}$

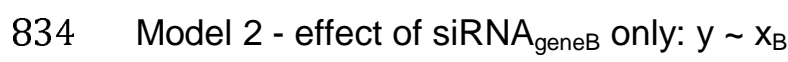

835 Model 3 for additive effect of both siRNAs (additive Gl): $y \sim x_{A}+x_{B}$

836 Model 4 - full model including genetic interaction (non-additive Gl): $y \sim x_{A}+x_{B}+x_{A}{ }^{*} X_{B}$.

838 To determine the best model explaining the data for each gene pair we used Schwarz's 839 Bayesian Information Criterion (BIC). BIC score was calculated for each model fitted to the data, 840 then the model with the lowest $\mathrm{BIC}$ value $\left(\mathrm{BIC}^{*}\right)$ was selected as the best-fitting model. Co841 knockdown effects of each gene pair were classified as aGls or naGls when model 3 or model 4 842 accordingly were defined to fit data best. Additionally, for the RNAi screen, we used the method 843 published by Raftery, 1995 to define the strength of evidence for the respective model to be 844 selected ${ }^{62}$. Namely, if the difference $(\triangle \mathrm{BIC})$ between the BIC value of the best fitting model (the 
845 model with the lowest BIC value) and the BIC value of any other model is bigger than 2, then it

846 would indicate a significant evidence for this model (with $\mathrm{BIC}^{\star}$ ) to truly represent the data. In

847 other words, if $\triangle \mathrm{BIC}>2$ then the model with lowest $\mathrm{BIC}$ value $\left(\mathrm{BIC}^{*}\right)$ was considered as the one

848 most correctly describing the data in comparison to other tested models. If the $\Delta \mathrm{BIC}<2$, then two

849 models were considered as possible alternatives for representing the dataset.

850 Secondly, to estimate statistical significance of gene interaction effect for each siRNA gene

851 combination, we calculated a $\mathrm{p}$-value from the t-value of the linear regression model term,

852 describing genetic interaction $\left(\beta_{A B}\right)$ as $p_{V a l}=2-2^{*} p_{n o r m}\left(a b s\left(t_{V a l}\right)\right)$. To correct for multiple

853 comparisons, the $p$-values were adjusted using the false discovery rate (fdr) method ${ }^{63}$, and the

854 fdr-corrected p-values $<10^{-2}$ were considered to correspond to significant Gls.

855

\section{RT-qPCR analysis}

857 Cell lysis and total RNA extraction was done using the RNease Mini Kit (Qiagen). Reverse858 transcription was performed with the SuperScript ${ }^{\mathrm{TM}}$ III First-Strand Synthesis SuperMix for RT859 qPCR (Invitrogen). RT-qPCR data was obtained from three biological replicates/siRNA 860 treatment. Primers can be provided upon request. For each siRNA treatment target mRNA was 861 normalized to that of GAPDH and compared to CTRL siRNA and the log2 of fold change $\left(2^{-\triangle \triangle C T}\right)$ 862 was calculated (see Figure S4). Results were considered as significant if $p$-values were below 8630.05 in a two-tailed Student's t-test.

864

\section{Immunocytochemistry and confocal microscopy}

866 Cells were transfected and cultured as described above then fixed with $3 \%$ paraformaldehyde 867 (PFA) at room temperature for $20 \mathrm{~min}$, washed with PBS, incubated with $30 \mathrm{mM}$ glycine for 5 min 868 and washed again with PBS. For LDLR staining cells were permeabilized with $0.05 \%$ Filipin III 869 (Sigma \#F4767) in 10\% FCS for $30 \mathrm{~min}$ at room temperature. Primary antibody: rabbit 870 monoclonal anti-LDLR (Fitzgerald \#20R-LR002) was diluted in 5\% FCS overnight at $4{ }^{\circ} \mathrm{C}$. 871 Secondary antibody: goat polyclonal goat anti-rabbit IgG Alexa 568 (Invitrogen \#A11011) was 872 diluted 1:400 in 5\% FCS. Fixed cells were imaged using a Zeiss LSM 780 confocal microscope 
bioRxiv preprint doi: https://doi.org/10.1101/2020.10.29.360818; this version posted October 30, 2020. The copyright holder for this preprint (which was not certified by peer review) is the author/funder, who has granted bioRxiv a license to display the preprint in perpetuity. It is made available under aCC-BY-NC-ND 4.0 International license.

\section{3 using a 63x/NA 1.4 oil objective.}

874 


\section{FIGURE LEGENDS}

\section{Figure 1}

877 PTV-burden tests in UK Biobank establish additive Gls for PCSK9-APOB and LPL-APOB

878 (a) Workflow of the study. 30 high-confidence candidate genes for Gl testing were chosen from 87918 GWAS regions associated with blood lipid traits or CAD risk based on colocalization analyses 880 with eQTL/pQTL signals and previously reported lipid-regulatory functions (see Methods). 881 Pairwise Gl analyses were conducted from three complementary datasets: protein-truncating 882 variants (PTVs) from exome sequencing in the UK Biobank; lipid/CAD GWAS lead SNPs; and 883 combinatorial RNAi (coRNAi) experiments in cells. Robust linear model fitting was used to 884 identify additive (aGl) and non-additive (naGl) Gls, and genetic and functional data were 885 integrated. (b) Gene-based PTV-burden analyses from 161,508 exomes identified an additive GI 886 (aGl) for LDL (and TC; not shown) between PSCK9 and APOB. (c) A suggestive non-additive GI 887 (naGl) for HDL (and TG; not shown) between PTVs in LPL and $A P O B$ was validated as aGl for 888 HDL, TG, and also LDL by replication analyses in an additional 79,462 UK Biobank exomes 889 (Table S6). n, number of carriers. (-), predicted loss-of-function due to PTVs.

890

891 Figure 2

892 Pairwise Gls between lipid and CAD GWAS lead SNPs in 387,033 UK Biobank participants

893 (a-e) Circos plots showing aGls (grey) and naGls (colored) between GWAS lead SNPs (blue) at 894 the 28 selected lipid/CAD loci (red) for the four tested lipid species and CAD. (f) Tests for Gls 895 between polygenic risk scores for the four lipid species and PTV-burden for each of the 30 lipid 896 genes identified a naGl between PTV-burden in LPL and the PRS for TG. PRS distribution 897 (mean \pm SD) for LPL-PTV carriers (pink) and non-carriers (blue) are plotted against mean 898 normalized residual TG levels. Each dot reflects mean TG levels at a respective percentile.

\section{Figure 3}

901 Combinatorial RNAi identifies pairwise Gls modulating cellular LDL-uptake 
902 (a) coRNAi screen workflow. Customized cell microarrays were generated by pairwise spotting of 903 siRNAs against two different candidate genes on 384 spots/array for solid-phase reverse siRNA

904 transfection of cultured HeLa cells. Cells challenged to internalize fluorescent-labelled LDL (Dil-

$905 \mathrm{LDL}$ ) over a period of $20 \mathrm{~min}$ were imaged on a high-content microscope. Integrated

906 fluorescence intensities for each cell individually were quantified by automated image analysis.

907 Averaged signal intensities per gene pair were tested for Gls in multiple replica experiments per

908 array. Gls suggested in the coRNAi screen as potentially non-additive were subsequently

909 validated in customized experiments using fluid-phase transfection. (b) Heatmap visualizing

910 median robust Z-score distribution upon coRNAi of 435 gene pairs assessed for their impact on

911 cellular LDL-uptake. Red, increase. Blue, decrease. CTRL (top row and first column) reflects the

912 relative impact on LDL-uptake when candidate genes were silenced individually $\left(\right.$ siRNA $_{\text {geneAorB }}+$

913 negative control siRNA). (c) 20 gene pairs validated as either buffering or synergistic naGls on

914 cellular LDL-uptake in independent replica experiments, sorted according to effect size.

915 Interaction Value (right graph) depicts the directionality and difference of the combined effect

916 versus single knockdown effects. (d-f) Selected examples of single gene (siRNA $A_{g e n e A}+$ negative

917 control siRNA) and gene pair (siRNA $\left.{ }_{\text {geneA }}+\mathrm{SiRNA}_{\text {geneB }}\right)$ siRNA knockdown effects on relative

918 fluorescently-labelled LDL (Dil-LDL) cellular uptake. CTRL, control siRNA. Boxplots represent

919 values between $25^{\text {th }}$ and $75^{\text {th }}$ percentile, whiskers indicate largest value within 1.5 times

920 interquartile range above $75^{\text {th }}$ percentile. Median value is highlighted in the boxplot as a

921 horizontal line. Dots represent robust Z-score values calculated for integrated Dil fluorescence

922 intensities per cell (see Methods). Scale bar=10 $\mu \mathrm{m}$.

923

\section{$924 \quad$ Figure 4}

925 Integrative analysis identifies pairwise Gls supported by both, genetic and functional data

926 Overlap of Gls identified through genetic analyses and coRNAi. Highlighted are gene pairs

927 identified through either PTV-SNP $(\mathbf{a}, \mathbf{b})$ or SNP-SNP $(\mathbf{c}, \mathbf{d})$ GI testing for which pairwise siRNA-

928 knockdown showed corresponding effects on cellular LDL-uptake, validating these Gls as either

$929 \mathrm{aGl}(\mathrm{a}, \mathrm{c})$ or naGI $(\mathrm{b}, \mathrm{d})$. (e) TOMM40 as an example for which, consistent with SNP-SNP 
930 analyses, siRNA knockdown revealed buffering naGls when jointly silenced with SORT1 (left

931 panel) or NCAN (right panel). Values on the graphs reflect robust Z-scores values calculated for

932 total intensity of Dil-LDL per cell averaged per image (see Methods). Boxplots represent values

933 between $25^{\text {th }}$ and $75^{\text {th }}$ percentile, whiskers indicate largest value within 1.5 times interquartile

934 range above $75^{\text {th }}$ percentile. Median value is highlighted in the boxplot as a horizontal line. Dots

935 represent robust Z-score values calculated for integrated Dil fluorescence intensities per cell

936 (see Methods). Scale bar $=10 \mu \mathrm{m}$. 
bioRxiv preprint doi: https://doi.org/10.1101/2020.10.29.360818; this version posted October 30, 2020. The copyright holder for this preprint

(which was not certified by peer review) is the author/funder, who has granted bioRxiv a license to display the preprint in perpetuity. It is made available under aCC-BY-NC-ND 4.0 International license.

\section{TABLES}

938 Table 1.

939 Non-additive Gls from pairwise PTV-burden and GWAS lead SNP-based GI testing in UK Biobank

940

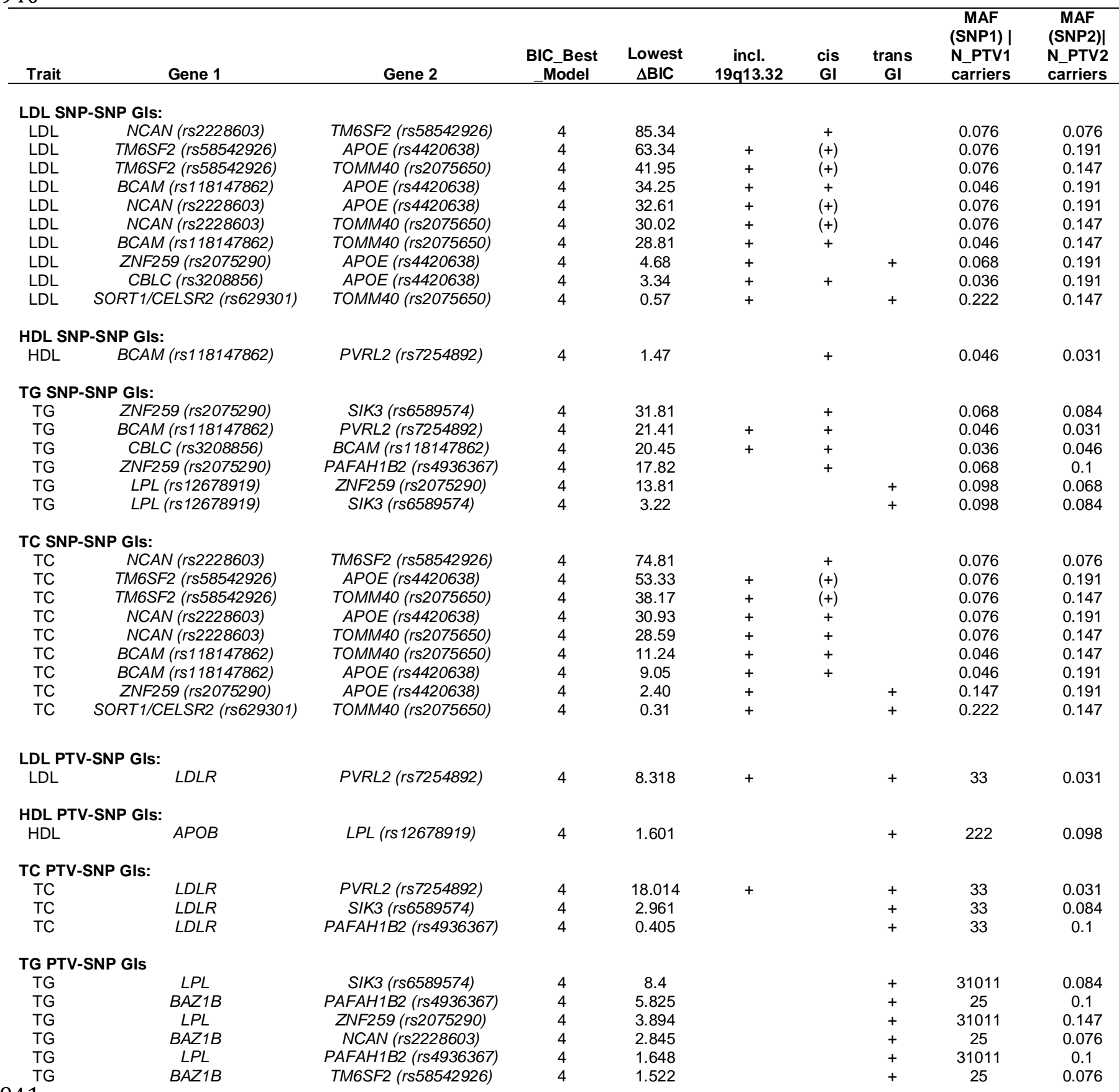

941

942 Non-additive genetic interactions (naGls) identified through GWAS lead SNP- and PTV-SNP-based GI analyses in the

943 UK Biobank as described in Methods. BIC, Bayesian Information Criterion. A lowest $\triangle B I C$ of "4" indicates interaction

944 model is most compatible with a non-additive interaction effect. MAF (minor allele frequency) estimates and numbers

945 of rare protein-truncating variant (PTV) carriers are based on genotypes from 387,033 and exomes, respectively, from

946161,508 unrelated UK Biobank participants of European ancestry. (+) indicates possible cis-effects of rs4420638 in

947 APOE on neighbouring genes on Chr.19q13.32. Trans GI indicates genes contributing to pairwise naGls are located

948 on different chromosomes. * represents data based on the replication study in additional 79,462 UK Biobank

949 participants. 
bioRxiv preprint doi: https://doi.org/10.1101/2020.10.29.360818; this version posted October 30, 2020. The copyright holder for this preprint (which was not certified by peer review) is the author/funder, who has granted bioRxiv a license to display the preprint in perpetuity. It is made available under aCC-BY-NC-ND 4.0 International license.

953 Table 2.

\begin{tabular}{|c|c|c|c|c|c|c|c|c|c|c|}
\hline \multicolumn{2}{|c|}{ GI Gene Pair } & \multirow[t]{2}{*}{$\begin{array}{l}\text { Interaction } \\
\text { type }\end{array}$} & \multicolumn{2}{|c|}{$\begin{array}{c}\text { BIC-based GI } \\
\text { testing }\end{array}$} & \multicolumn{3}{|c|}{ RLMF-based GI testing } & \multicolumn{3}{|c|}{ Validation RNAi GI testing } \\
\hline Gene1 & Gene2 & & $\begin{array}{c}\text { Lowest } \\
\Delta \mathrm{BIC}\end{array}$ & $\begin{array}{c}\text { Best } \\
\text { Model }\end{array}$ & $\begin{array}{l}\text { Robust } \\
\text { Zscore }\end{array}$ & $\begin{array}{l}\text { Interaction } \\
\text { Value }\end{array}$ & pVal(fdr) & $\begin{array}{l}\text { Robust } \\
\text { Zscore }\end{array}$ & $\begin{array}{l}\text { Interaction } \\
\text { Value }\end{array}$ & pVal (fdr) \\
\hline$A P O B$ & HMGCR & \multirow{6}{*}{ 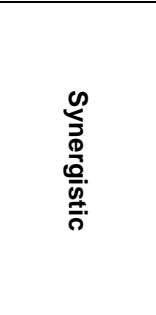 } & 4.62 & 2 & 2.8 & 2.18 & 4.05E-03 & 3.33 & 0.97 & $1.53 \mathrm{E}-03$ \\
\hline HAVCR1 & $L D L R$ & & 4.31 & 4 & -2.18 & -1.32 & $1.68-02$ & -2.24 & -1.24 & $1.81 \mathrm{E}-10$ \\
\hline$L D L R$ & NCAN & & 2.36 & 4 & -1.86 & -1.28 & 7.64E-03 & -1.23 & -2.22 & $2.20 \mathrm{E}-11$ \\
\hline MYBPHL & SIK3 & & 2.27 & 4 & 2.17 & 1.59 & $6.59 \mathrm{E}-03$ & 2.3 & 0.96 & 7.91E-03 \\
\hline PAFAH1B1 & SIK3 & & 4.49 & 4 & 1.62 & 1.79 & $2.52 \mathrm{E}-03$ & 3.3 & 2.44 & 8.37E-12 \\
\hline PCSK9 & TMEM57 & & 3.82 & 4 & 1.46 & 1.76 & $2.37 \mathrm{E}-03$ & 3.21 & 2.44 & $3.74 \mathrm{E}-11$ \\
\hline$B C A M$ & LDLRAP1 & \multirow{14}{*}{ 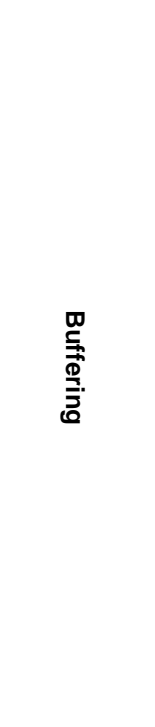 } & 4.70 & 4 & -0.4 & -1.83 & $3.58 \mathrm{E}-04$ & -0.05 & -0.7 & $5.20 \mathrm{E}-03$ \\
\hline CELSR2 & $L P L$ & & 0.04 & $0 / 2$ & -1.4 & -1.43 & $7.53 \mathrm{E}-03$ & -0.11 & -0.86 & $8.70 \mathrm{E}-05$ \\
\hline CXCL12 & PAFAH1B1 & & 5.47 & 4 & -2.17 & -1.78 & 3.69E-04 & 1.32 & -1.92 & $9.84 \mathrm{E}-13$ \\
\hline HAVCR1 & LDLRAP1 & & 13.70 & 4 & -0.56 & -2.16 & 1.03E-05 & 0.39 & -0.73 & $5.40 \mathrm{E}-03$ \\
\hline HAVCR1 & $M L X I P L$ & & 13.55 & 4 & -0.63 & -2.31 & $1.03 \mathrm{E}-05$ & -0.12 & -1.2 & 2.06E-05 \\
\hline HAVCR1 & SEZ6L & & 8.54 & 4 & -1.19 & -1.91 & 3.39E-04 & -0.67 & -0.77 & $2.90 \mathrm{E}-04$ \\
\hline HAVCR1 & SORT1 & & 11.66 & 4 & -2.09 & -2.24 & $9.34 \mathrm{E}-05$ & -0.63 & -0.58 & $1.98 \mathrm{E}-03$ \\
\hline$L D L R$ & LDLRAP1 & & 10.06 & 4 & -2.44 & -1.86 & $9.34 \mathrm{E}-05$ & -2.49 & -0.92 & $2.36 \mathrm{E}-10$ \\
\hline LDLR & $M L X I P L$ & & 5.79 & 4 & -2.03 & -1.49 & 2.97E-03 & -2.26 & -0.78 & 4.19E-11 \\
\hline LDLRAP1 & SORT1 & & 8.13 & 4 & -1.11 & -1.65 & $2.57 \mathrm{E}-03$ & -1.09 & -0.95 & $3.89 \mathrm{E}-06$ \\
\hline$M L X I P L$ & TOMM40 & & 18.73 & 4 & -2.14 & -2.67 & $1.03 \mathrm{E}-05$ & -1.94 & -0.85 & $3.82 \mathrm{E}-08$ \\
\hline NCAN & SEZ6L & & 5.08 & 4 & -0.59 & -1.6 & $5.54 \mathrm{E}-03$ & -0.08 & -1.56 & $7.94 \mathrm{E}-12$ \\
\hline $\operatorname{NCAN}^{*}$ & TOMM40* & & 2.76 & 4 & -1.4 & -1.56 & 7.17E-03 & -1.49 & -1.34 & $1.75 \mathrm{E}-08$ \\
\hline SORT1* & TOMM40* & & 5.80 & 0 & 0.77 & 1.84 & $3.90 \mathrm{E}-03$ & -2.48 & 1.77 & $0.00 \mathrm{E}+00$ \\
\hline
\end{tabular}

954 Pairwise Gls identified and validated through coRNAi to impact LDL-uptake into cells

955

956 Gene pairs identified and independently validated by combinatorial RNAi (coRNAi) as impacting the uptake of

957 fluorescent-labelled LDL into cells in a non-additive manner. Both, BIC, Bayesian Information Criterion and RLMF,

958 Robust Linear Model Fitting were applied for analysis of coRNAi-based Gl-testing as described in Methods. *, gene

959 pairs that both, genetic and coRNAi Gl-testing identify as naGls.

961

962

963

964

965

966

967

968

969

970

971

972

973

974

975

976 
bioRxiv preprint doi: https://doi.org/10.1101/2020.10.29.360818; this version posted October 30, 2020. The copyright holder for this preprint (which was not certified by peer review) is the author/funder, who has granted bioRxiv a license to display the preprint in perpetuity. It is made available under aCC-BY-NC-ND 4.0 International license.

978

979

980

981

982

983

984

985

986

987

988

989 


\section{Figure 1}

a

bioRxiv preprint doi: https://doi.org/10.1101/2020.10.29.360818; this version posted October 30, 2020. The copyright holder for this preprint

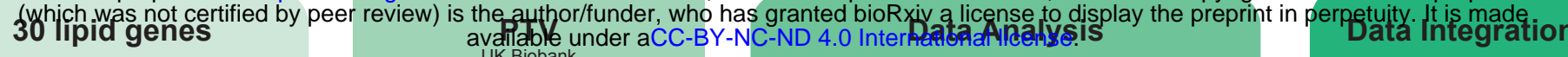
161,508 exomes

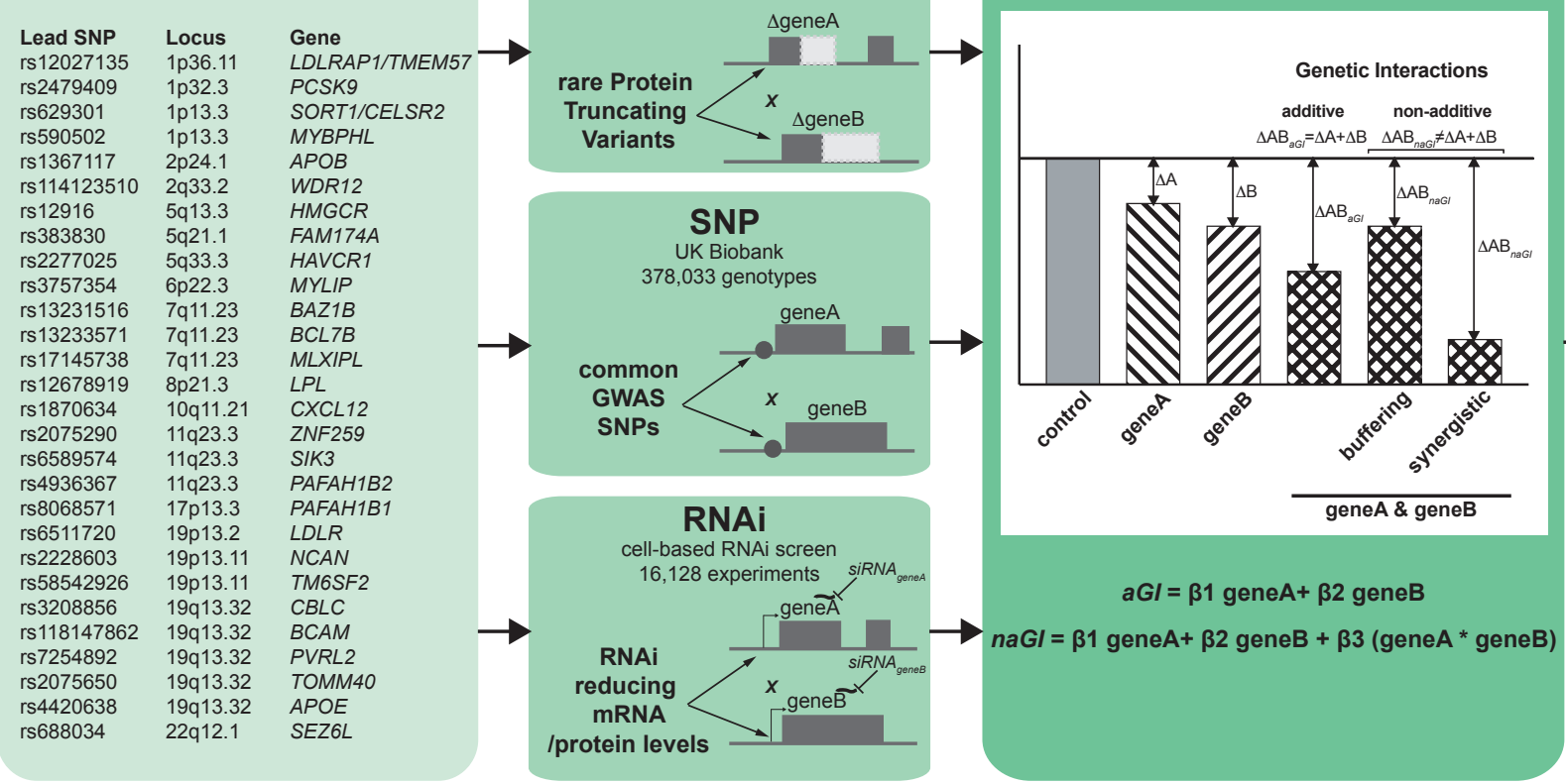

identification of overlapping aG/s and naG/s

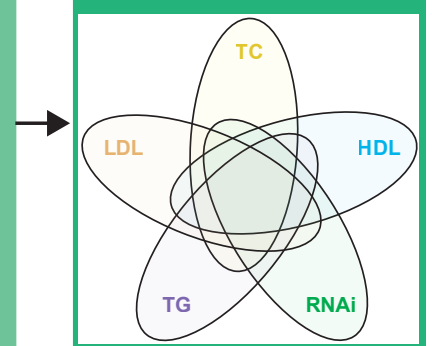

b

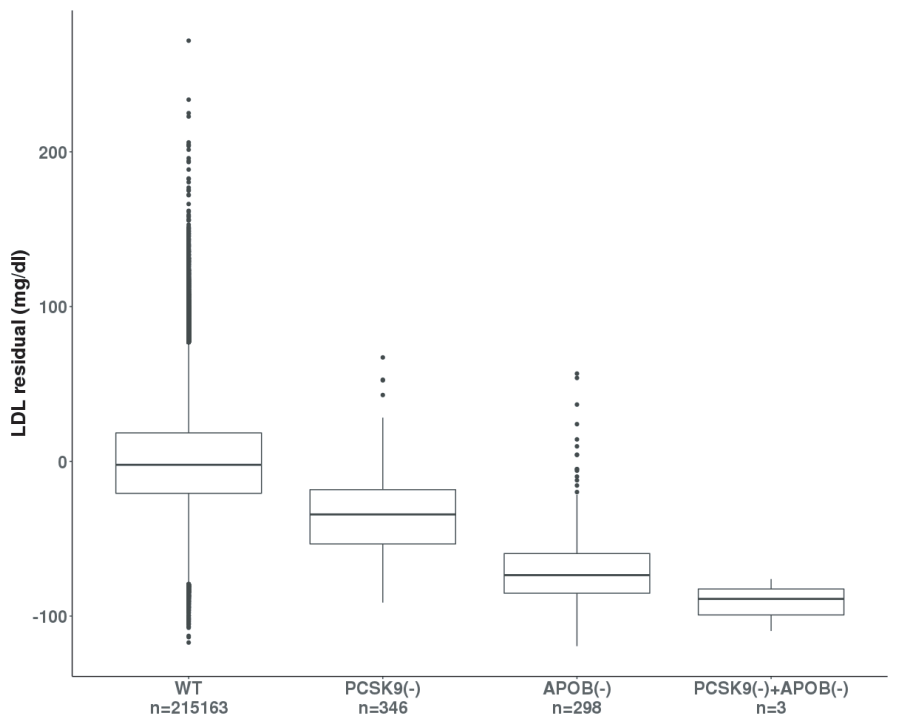

C

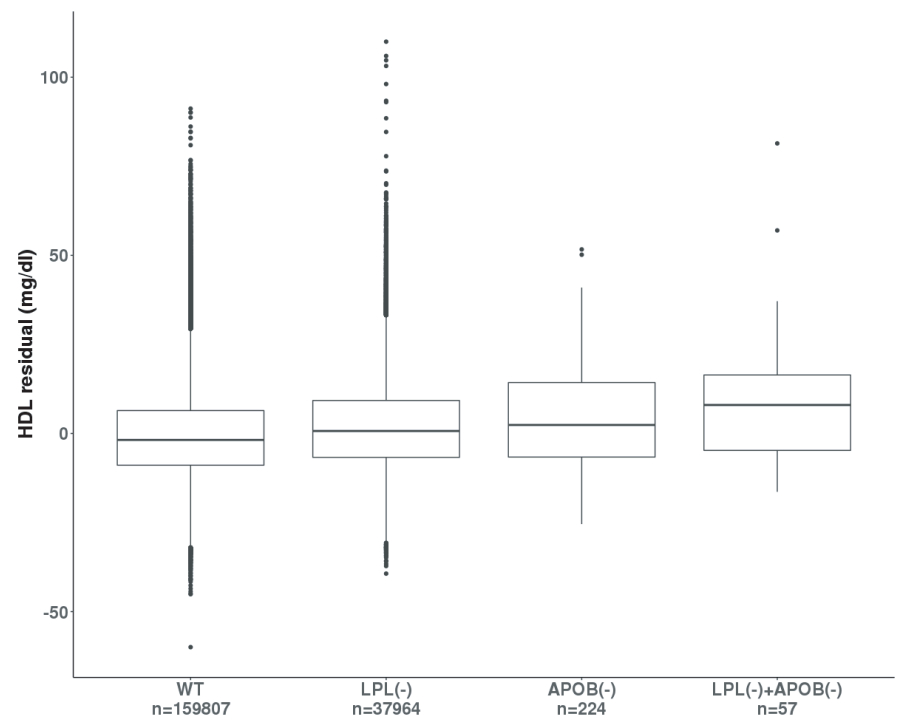


Figure 2

bioRxiv preprint doi: https:/doi.or / $/ 10.1101 / 2020.10 .29 .360818$; this version posted October 30, 2020 (which was not certified by peer review) is sthe author/funder, who has granted bioRxiv a license to display the pfeprint in perpetuity. It is made

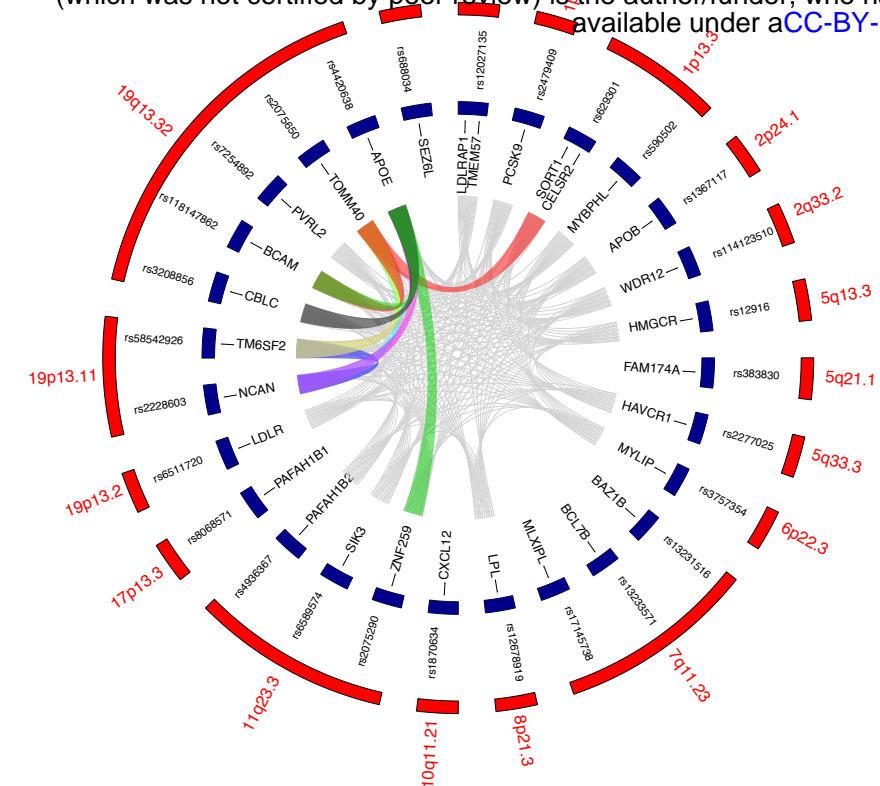

C

TG

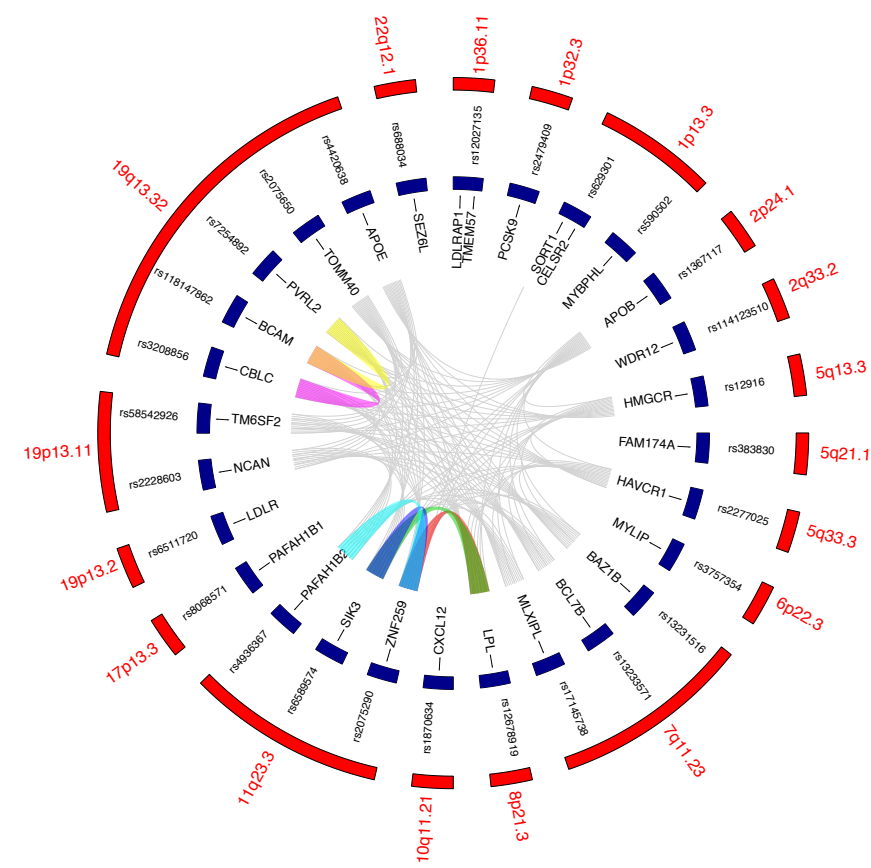

e

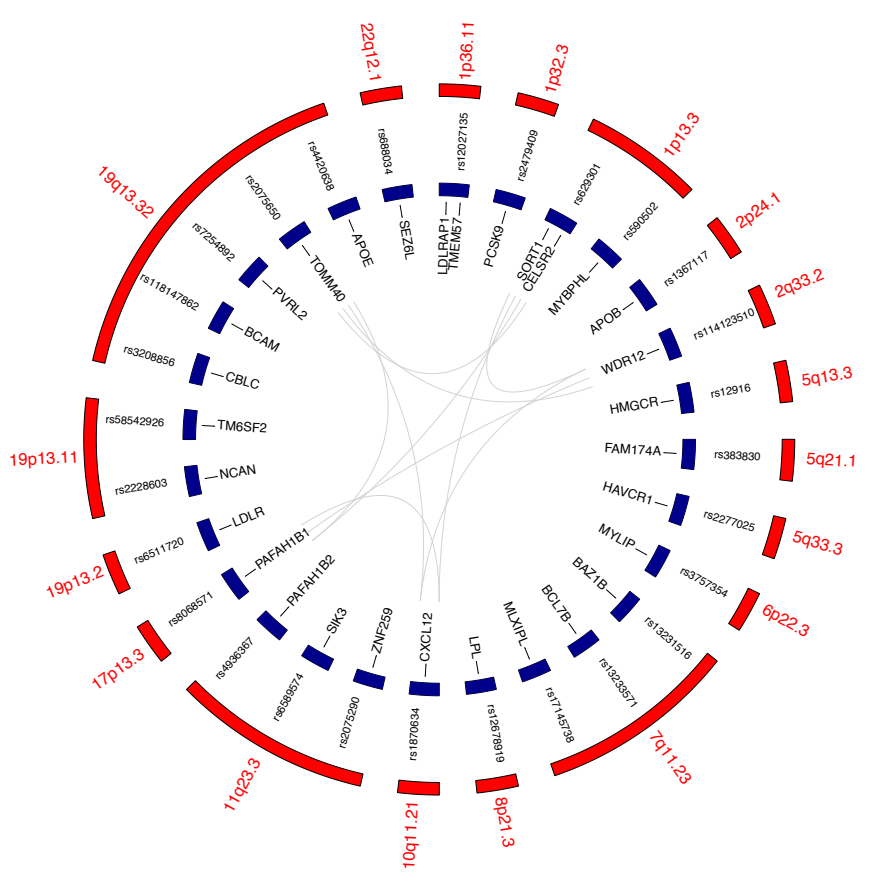

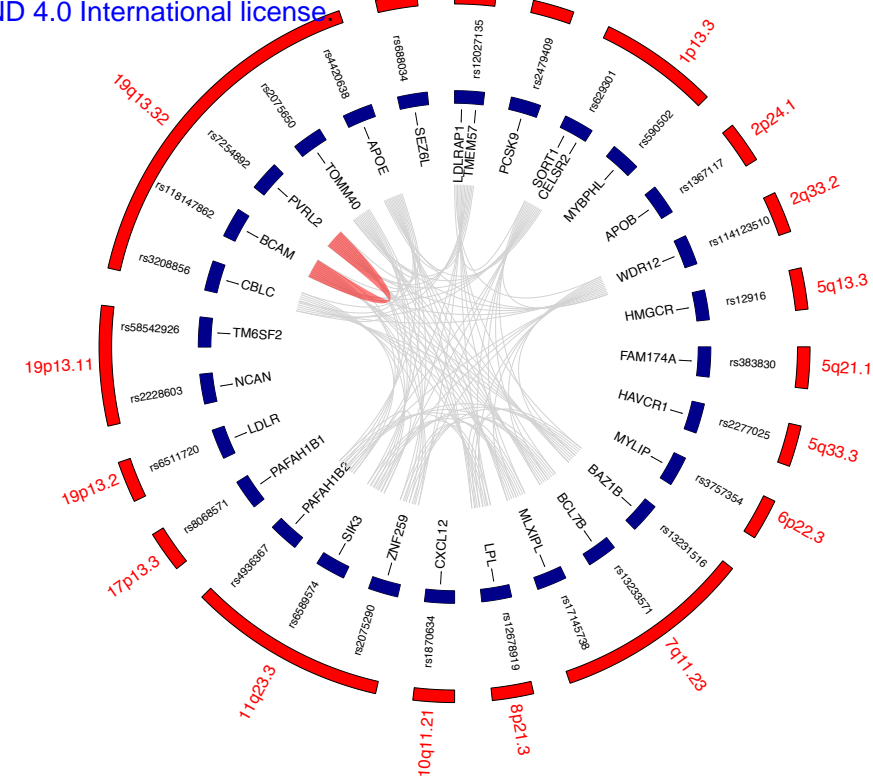

d

TC

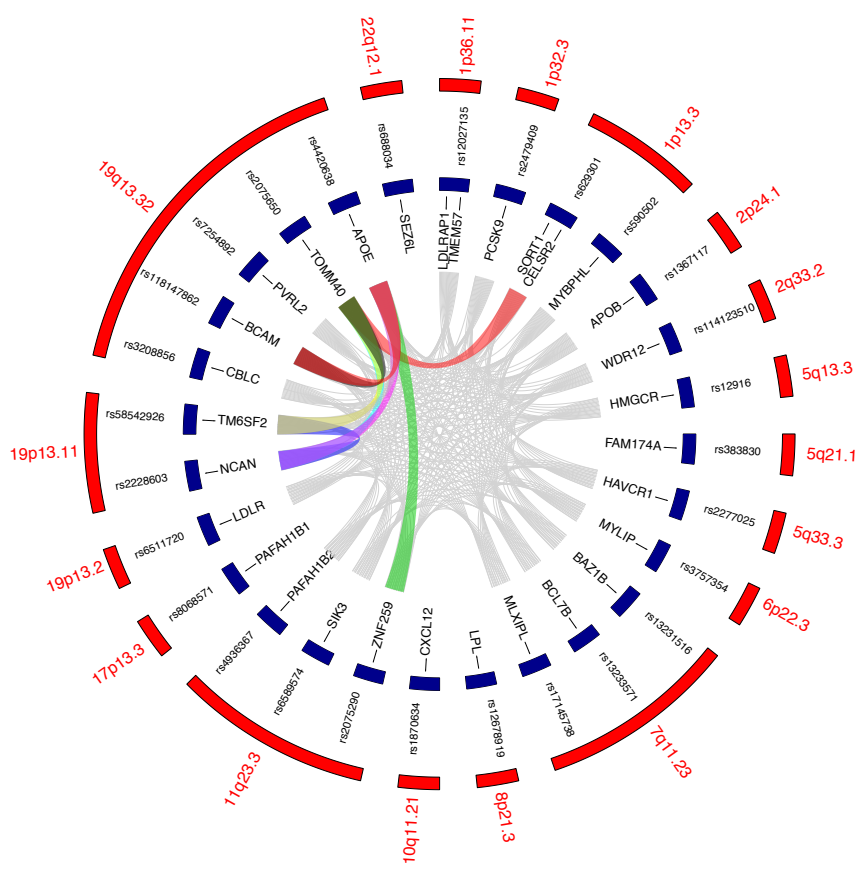

f

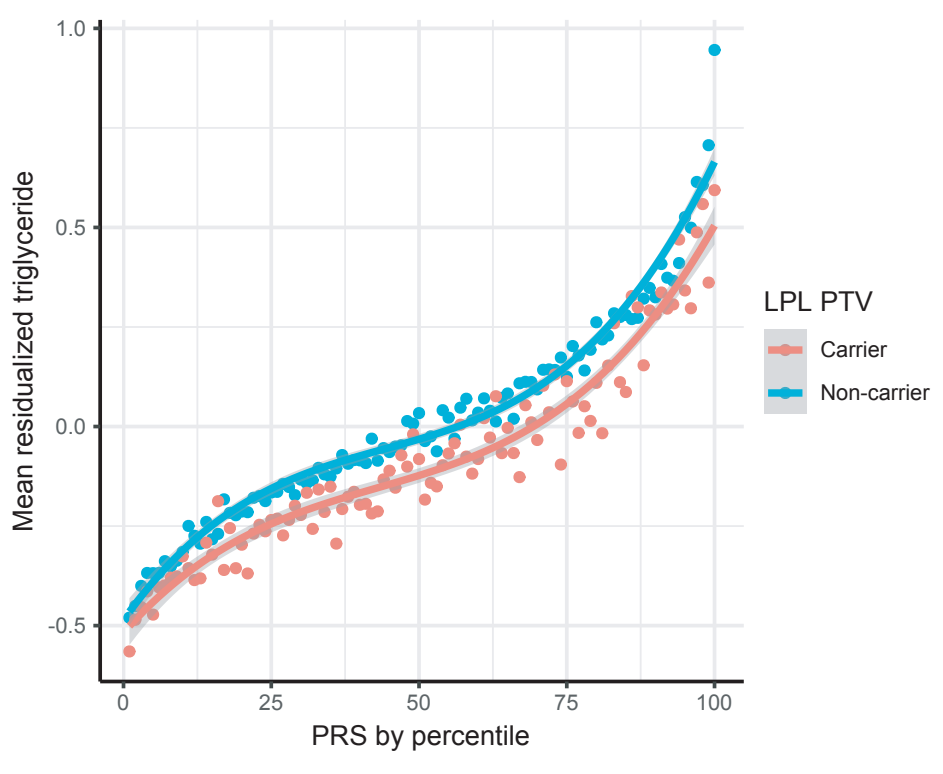


Figure 3

a

bioRxiv preprint doi: https://doi.org/10.1101/2020.10.29.360818; this version posted October 30, 2020, The copyright holder for this preprint (which was not certified by peer review) is the author/funder, who has granted bioRxiv a ticense to display the preprint in perpetuity. It is made

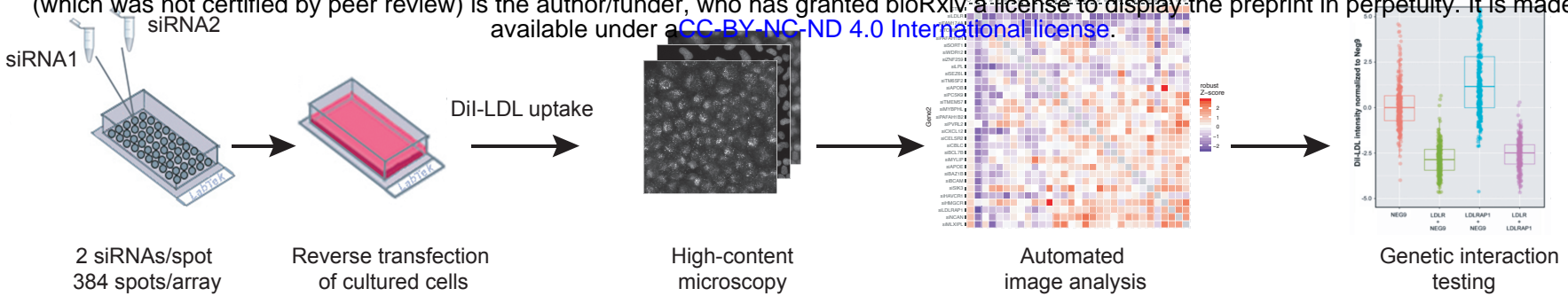

b

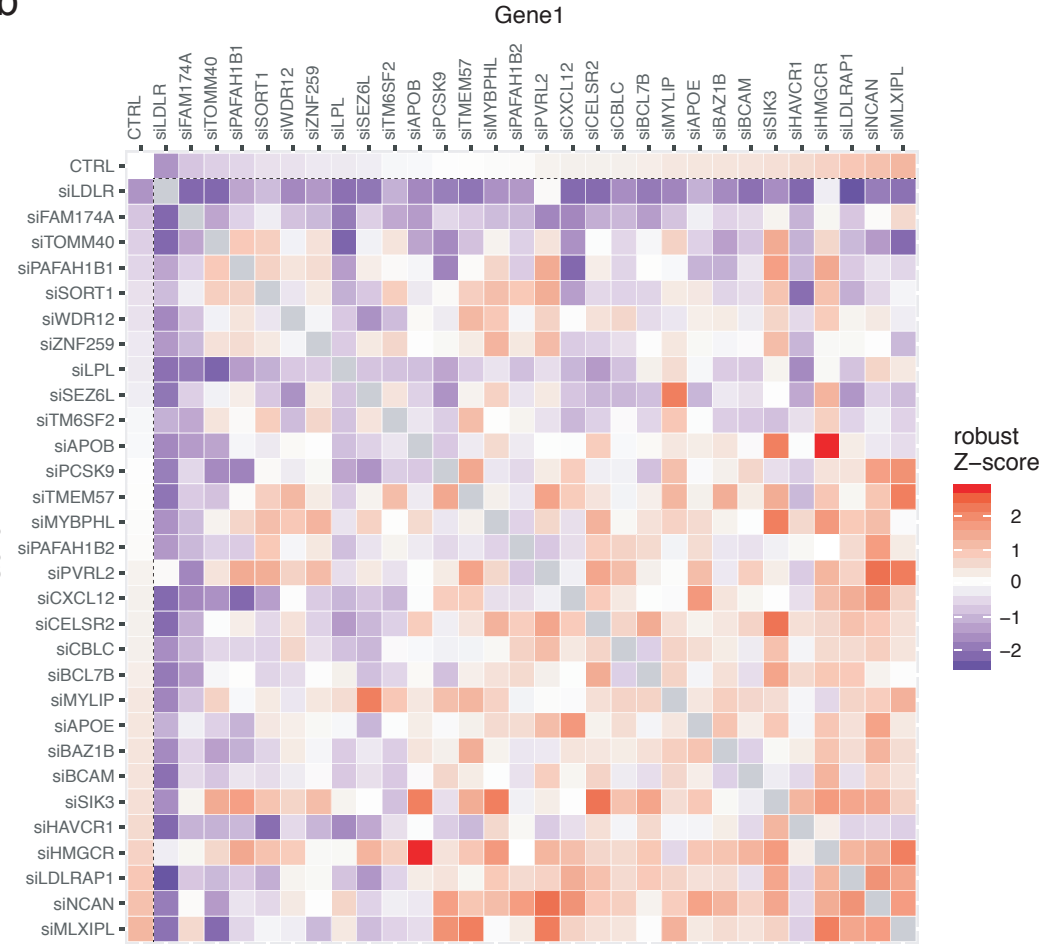

C

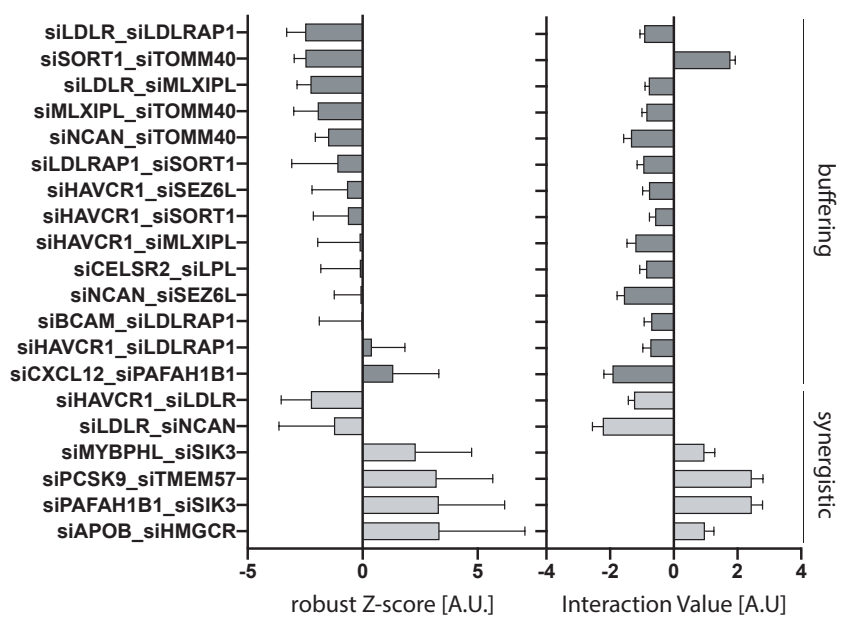

e
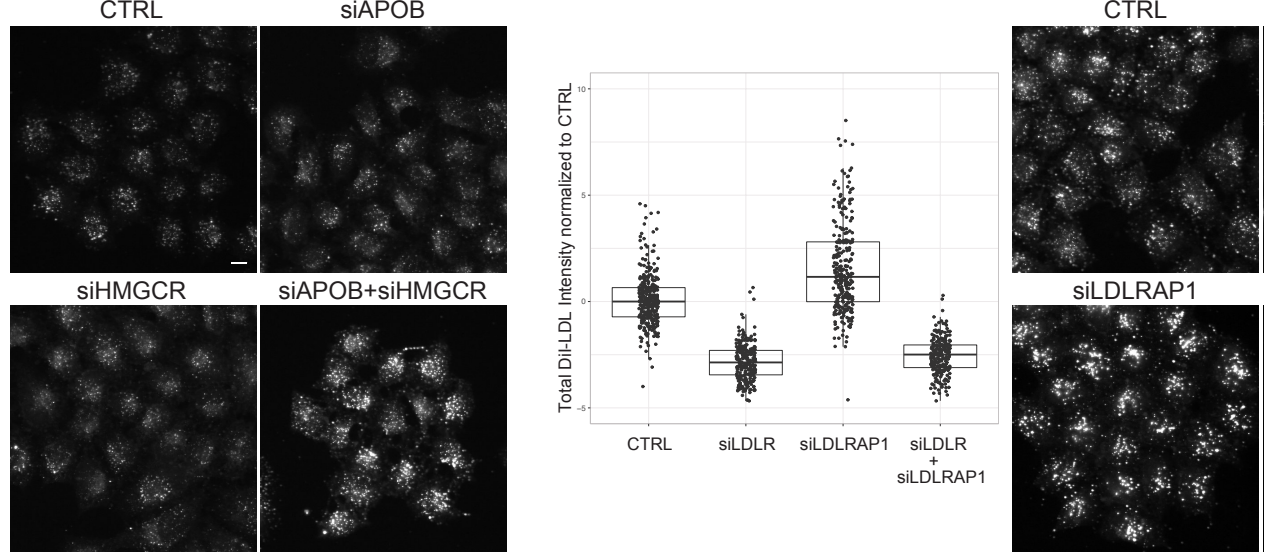

SiLDLRAP1

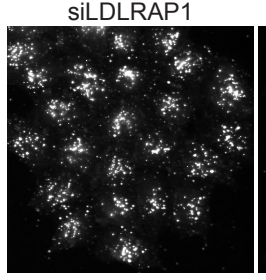

SiLDLR+siLDLR

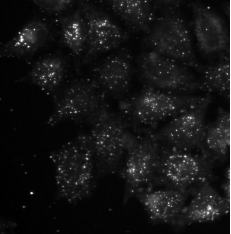

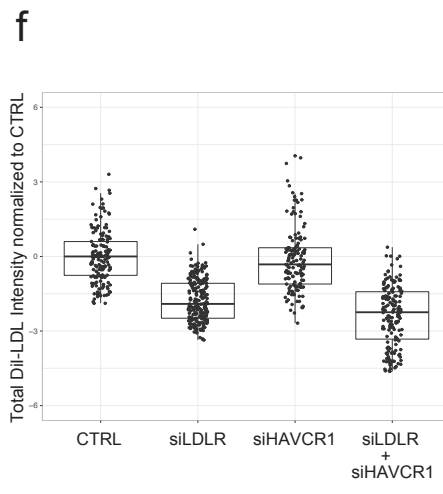

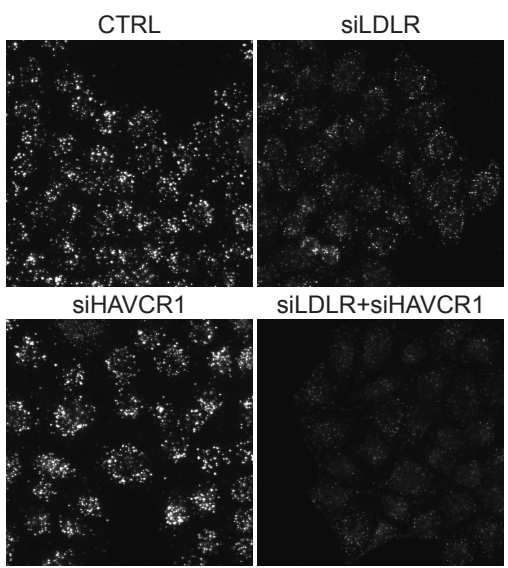




\section{Figure 4}

a bioRxiv preprint doi: https://doisrg/10.1101/2020.10.29.360818; this versign posted October 30, 2020 Ihe Gopyright holder for this preprint

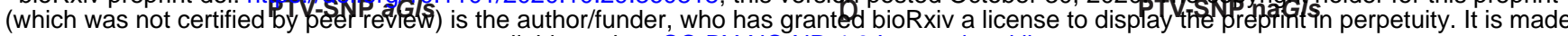

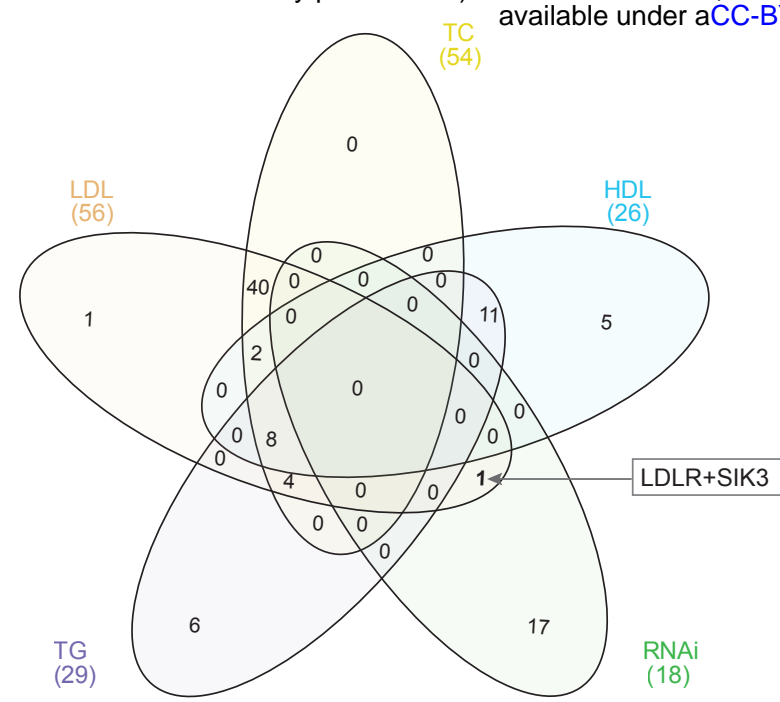

C

SNP-SNP aGIs
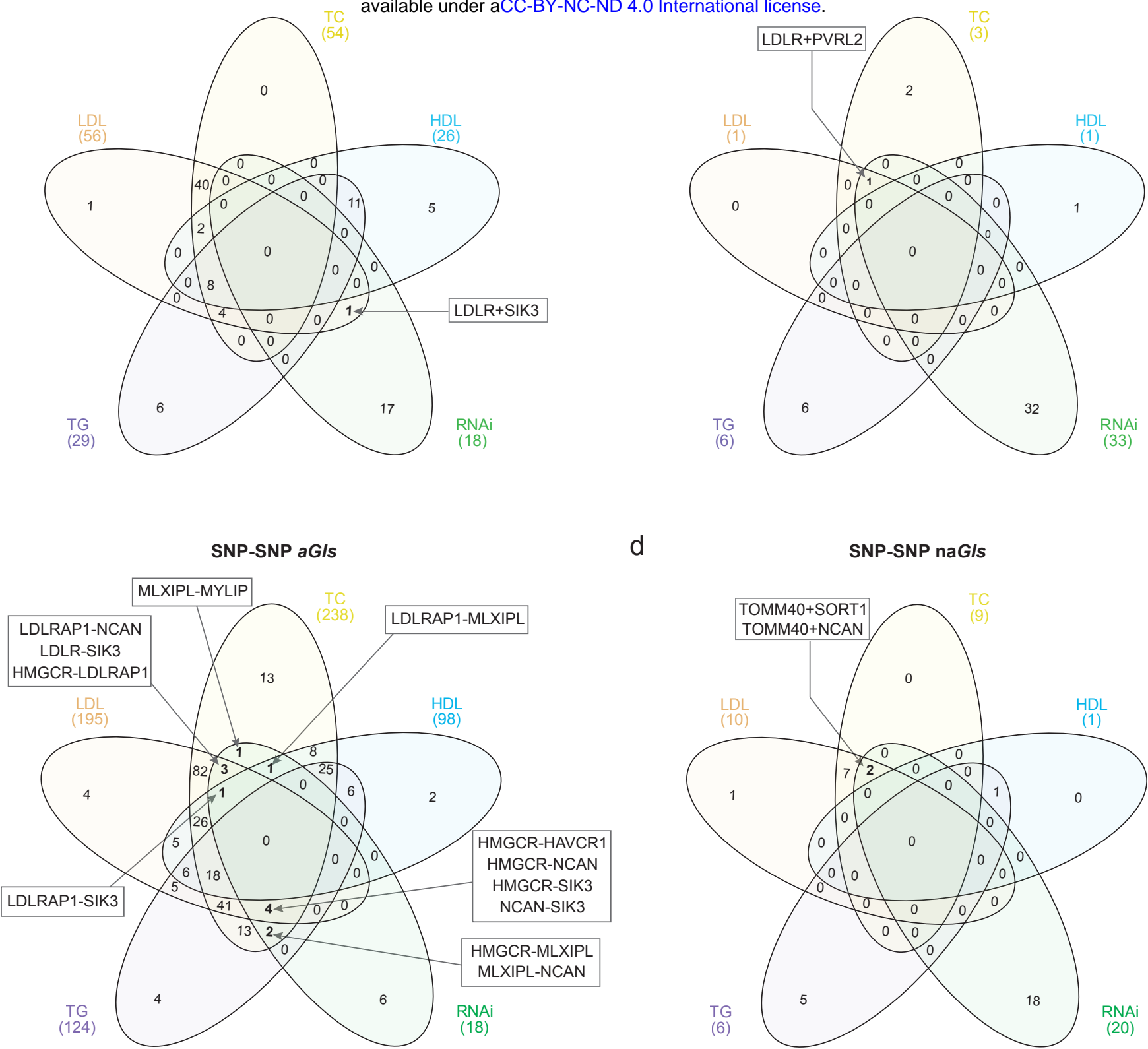

d

SNP-SNP naGls

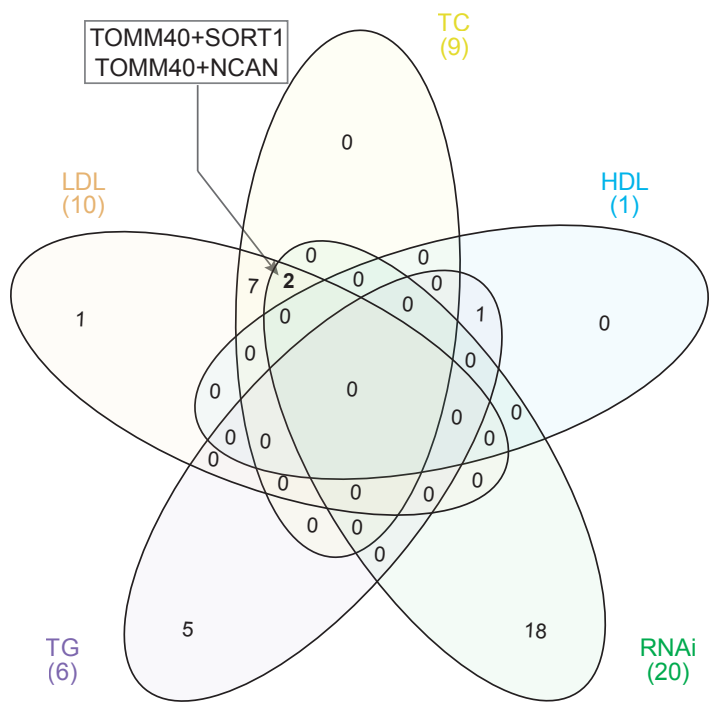

e
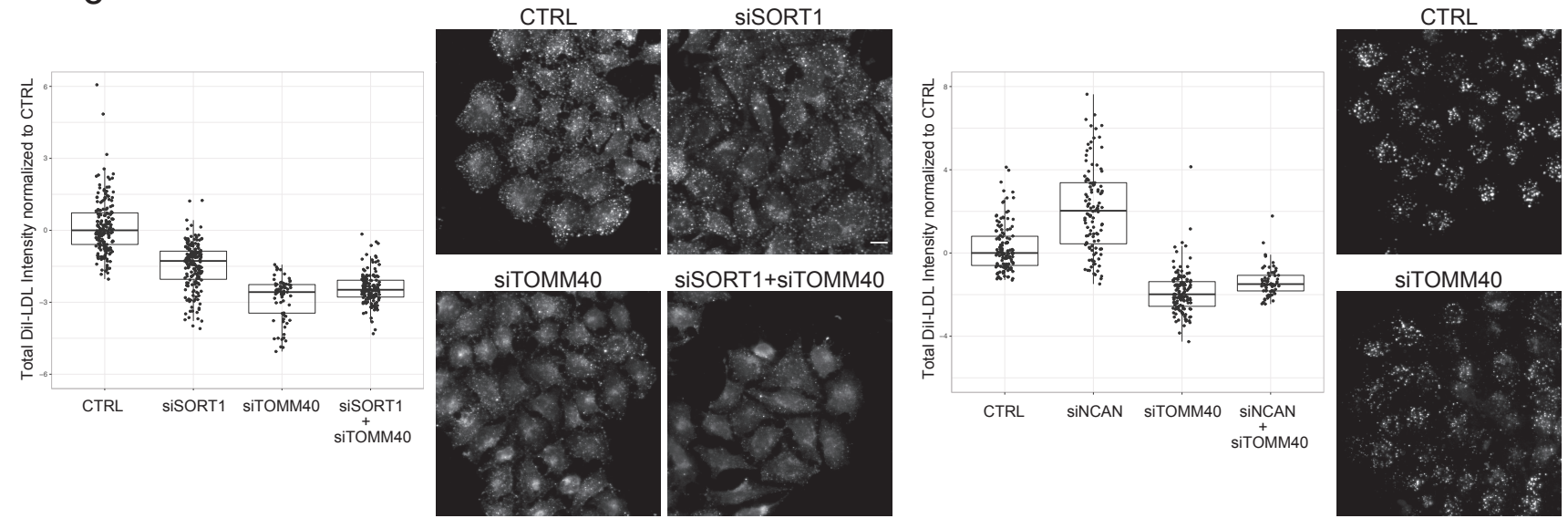

SiNCAN

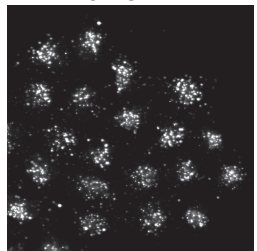

SiNCAN+siTOMM40

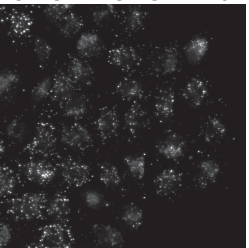

